Groups Geom. Dyn. 8 (2014), 97-134

DOI $10.4171 / \mathrm{GGD} / 218$
Groups, Geometry, and Dynamics

(C) European Mathematical Society

\title{
Rips induction: index of the dual lamination of an $\mathbb{R}$-tree
}

\author{
Thierry Coulbois and Arnaud Hilion
}

\begin{abstract}
Let $T$ be a $\mathbb{R}$-tree in the boundary of the Outer Space $\mathrm{CV}_{N}$, with dense orbits. The $Q$-index of $T$ is defined by means of the dual lamination of $T$. It is a generalisation of the Poincaré-Lefschetz index of a foliation on a surface. We prove that the $\mathcal{Q}$-index of $T$ is bounded above by $2 N-2$, and we study the case of equality. The main tool is to develop the Rips machine in order to deal with systems of isometries on compact $\mathbb{R}$-trees.

Combining our results on the $\mathcal{Q}$-index with results on the classical geometric index of a tree, developed by Gaboriau and Levitt, we obtain a beginning classification of trees.
\end{abstract}

Mathematics Subject Classification (2010). 20E08, 20E05, $20 \mathrm{~F} 65$.

Keywords. $\mathbb{R}$-trees, Outer Space, Rips machine.

\section{Introduction}

The space of minimal, free and discrete actions by isometries of the free group $\mathbb{F}_{N}$ of finite rank $N \geq 2$ on $\mathbb{R}$-trees has been introduced by Culler and Vogtmann [CV86]. Its projectivization is called Outer Space, and we denote it by $\mathrm{CV}_{N}$. It has a Thurstonboundary $\partial \mathrm{CV}_{N}$, which gives rise to a compactification $\overline{\mathrm{CV}}_{N}=\mathrm{CV}_{N} \cup \partial \mathrm{CV}_{N}$. Elements of this compact space $\overline{\mathrm{CV}}_{N}$ are projective classes [T] of minimal, very small actions by isometries of the free group $\mathbb{F}_{N}$ on $\mathbb{R}$-trees $T$ (see [CL95] and [BF95]). The reader will find a survey on Outer Space in [Vog02].

In this article, we introduce and study the $\mathcal{Q}$-index $\operatorname{ind}_{\mathcal{Q}}(T)$ of $\mathbb{R}$-trees $T$ in $\partial \mathrm{CV}_{N}$ with dense orbits. The $\mathcal{Q}$-index of an $\mathbb{R}$-tree (see Section 5.2) naturally extends the Poincaré-Lefschetz index of a foliation on a surface as explained below. The main result of our paper regarding this $\mathcal{Q}$-index is:

Theorem 5.3. Let $T$ be an $\mathbb{R}$-tree with a very small, minimal action of $\mathbb{F}_{N}$ by isometries with dense orbits. Then

$$
\operatorname{ind}_{\mathcal{Q}}(T) \leq 2 N-2 \text {. }
$$

We also characterize the case of equality, see Section 4.5. 
This $\mathcal{Q}$-index characterizes dynamical properties of trees. Using it together with the geometric index introduced by Gaboriau and Levitt [GL95] we obtain a classification of trees.

Theorem 5.3 already has several important consequences.

First, it answers a question of Levitt and Lustig, see Remark 3.6 in [LL03], on the finiteness of the fibres of the map $\mathcal{Q}$ (see below).

In our paper [CH10], we obtain a qualitative classification of fully irreducible outer automorphisms of free groups which extends that of Handel and Mosher [HM07] and of Guirardel [Gui05]. The key point is to interpret the index of an iwip automorphism [GJLL98] as the $\mathcal{Q}$-index of its repelling tree in $\partial \mathrm{CV}_{N}$.

In our paper with P. Reynolds [CHR11] we define an induction analogous to Rauzy-Veech for trees in $\partial \mathrm{CV}_{N}$. As we are working with systems of isometries on compact trees, Theorem 5.3 is crucially used to ensure that there are points where to start the splitting procedure.

1.1. Measured foliations on surfaces. Let $\Sigma$ be a surface of negative Euler characteristic, $\chi(\Sigma)<0$, with a measured foliation $\mathscr{F}$ (see [FLP79]). The foliation lifts to a measured foliation $\tilde{\mathcal{F}}$ of the universal cover $\tilde{\Sigma}$ of $\Sigma$. The space of leaves of $\tilde{\mathcal{F}}$ is an $\mathbb{R}$-tree $T$ : the distance in the tree $T$ is given by the transverse measure of the foliation $\tilde{\mathcal{F}}$ (see for instance Chapter 11 of [Kap01]). This tree comes with a small action of the fundamental group of $\Sigma$. When $\Sigma$ has non-empty boundary, its fundamental group is a free group $\mathbb{F}_{N}$ and $T$ defines an element of $\overline{\mathrm{CV}}_{N}$. The foliation $\mathcal{F}$ has $k$-prong singularities which give rise to branch points of valence $k$ in the tree. Locally the picture is as in Figure 1. We say that the foliation and the tree are dual to each other.

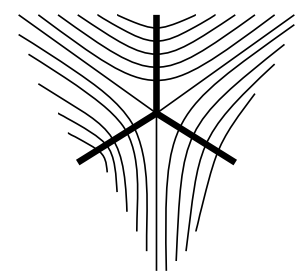

Figure 1. 3-prong singularity and the transverse tree (in bold). The local Poincaré-Lefschetz index is $-\frac{1}{2}$ and the local contribution to the $\mathcal{Q}$-index is 1 .

A local index can be defined for each singularity $P$ : the Poincaré-Lefschetz index. In this paper, we rather consider minus two times this index: ind $(P)$ can be defined as the number of half-leaves reaching the singular point, minus two. Alternatively, $\operatorname{ind}(P)$ is the valence of the corresponding point in the tree, minus two. Adding-up over all singular points in $\Sigma$, we obtain the (global) index of the foliation, which turns out to be equal to $-2 \chi(\Sigma)\left(=2 N-2\right.$ when $\left.\pi_{1}(\Sigma)=\mathbb{F}_{N}\right)$. 




Figure 2. Vertical foliation of the mapping torus of an interval exchange transformation. This foliation has two singularities (in bold), each of Poincaré-Lefschetz index $-\frac{1}{2}$ and local $\mathcal{Q}$ index 1 . The $\mathcal{Q}$-index of the foliation is 2 . The surface is a torus with one boundary component: its Euler characteristic is -1 .

Interval exchange transformations provide examples of such foliated surfaces. Indeed, the mapping torus of an interval exchange transformation is a surface (with boundary), naturally foliated by the vertical direction, as in Figure 2. The transverse measure of the foliation is given by the Lebesgue measure of the interval. We define, in this case, the index of the interval exchange transformation as the index defined above for this foliation and its dual $\mathbb{R}$-tree.

1.2. Geometric trees. This surface situation has been generalized (see for instance [Bes02]). Let us consider a finite family of partial isometries of an interval (or a finite number of intervals, or even a finite tree). The suspension of these partial isometries gives rise to a 2-complex $B$ (which is not a surface in general), naturally foliated by the vertical direction. As previously, the foliation can be lifted to the universal cover of $B$, and the space of leaves of this foliation is an $\mathbb{R}$-tree with an action of $\pi_{1}(B)$ by isometries. A tree obtained by such a construction is called a geometric tree. In this situation, we can define two local indices: one for the tree, using the valence of branch points, and one for the foliation, using the number of ends of singular leaves. We would like to stress that, contrary to the case of a foliation on a surface, these two indices do not agree, not even locally.

The first index has been introduced by Gaboriau and Levitt [GL95]. In this paper we call it the geometric index, and denote it by $\operatorname{ind}_{\mathrm{geo}}(T)$. It is defined using the valence of the branch points, of the $\mathbb{R}$-tree $T$, with an action of the free group by isometries:

$$
\operatorname{ind}_{\mathrm{geo}}(T)=\sum_{[P] \in T / \mathbb{F}_{N}} \operatorname{ind}_{\mathrm{geo}}(P) .
$$

where the local index of a point $P$ in $T$ is

$$
\operatorname{ind}_{\text {geo }}(P)=\#\left(\pi_{0}(T \backslash\{P\}) / \operatorname{Stab}(P)\right)+2 \operatorname{rank}(\operatorname{Stab}(P))-2 .
$$


Gaboriau and Levitt [GL95] proved that the geometric index of a geometric tree is equal to $2 N-2$ and that for any tree in the compactification of Outer Space $\overline{\mathrm{CV}}_{N}$ the geometric index is bounded above by $2 N-2$. Moreover, they proved that the trees in $\overline{\mathrm{CV}}_{N}$ with geometric index equal to $2 N-2$ are precisely the geometric trees.

The second index is defined from the number of ends of singular leaves. To our knowledge it has never been studied in its own right, although Gaboriau [Gab96] gives a lot of relevant insights. In particular Gaboriau [Gab96], Theorem VI.1, gives partial results to bound this index.

1.3. The $\mathcal{Q}$-index of an $\mathbb{R}$-tree. Let $T$ be an $\mathbb{R}$-tree in the boundary of Outer Space with dense orbits. We denote by $\bar{T}$ its metric completion, $\partial T$ its Gromov-boundary at infinity. The set $\widehat{T}=\bar{T} \cup \partial T$ equipped with the observers' topology (a slight weakening of the metric topology, see [CHL07]) is a compact set denoted $\widehat{T}^{\text {obs }}$.

Let $P$ be a point in $T$. The map $\mathcal{Q}: \partial \mathbb{F}_{N} \rightarrow \widehat{T}^{\text {obs }}$ is the unique continuous extension (see [CHL07]) of the map

$$
\mathbb{F}_{N} \rightarrow T, \quad u \mapsto u \cdot P .
$$

It does not depend on the choice of the point $P$.

The map $\mathcal{Q}$ can be easily understood in the special case of a tree $T$ dual to a foliation on a 2-complex $B$. Each leaf of the foliation of $\tilde{B}$ is a point of the dual tree $T$. A half-leaf of the foliation of $\tilde{B}$ defines a point $X \in \partial \mathbb{F}_{N}=\partial \pi_{1}(B)$ and $\mathcal{Q}(X)$ is the point of $T$ defined by the leaf.

The general definition of the $\mathcal{Q}$-index of the tree $T$ is given as follows:

$$
\operatorname{ind}_{\mathcal{Q}}(T)=\sum_{[P] \in \widehat{T} / \mathbb{F}_{N}} \max \left(0 ; \operatorname{ind}_{\mathcal{Q}}(P)\right) .
$$

where the local index of a point $P$ in $T$ is:

$$
\operatorname{ind}_{\mathcal{Q}}(P)=\#\left(\mathcal{Q}^{-1}(P) / \operatorname{Stab}(P)\right)+2 \operatorname{rank}(\operatorname{Stab}(P))-2 .
$$

Levitt and Lustig [LL03] proved that points in $\partial T$ have exactly one pre-image by Q (see Proposition 5.2). Thus, only points in $\bar{T}$ contribute to the $\mathcal{Q}$-index of $T$.

Our main result states that the $\mathcal{Q}$-index of an $\mathbb{R}$-tree in the boundary of Outer Space is bounded above by $2 N-2$. This answers the question of Levitt and Lustig ([LL03], Remark 3.6) whether the map $\mathcal{Q}: \partial \mathbb{F}_{N} \rightarrow \widehat{T}$ has finite fibers (in the case where the action is free).

In [CHL08] the dual lamination of $T$ is defined: it is the set of pairs $(X, Y)$ of distinct points in the boundary $\partial \mathbb{F}_{N}$ such that $\mathcal{Q}(X)=\mathcal{Q}(Y)$. More conceptually, the $Q$-index of $T$ can indeed be understood in a more general context as that of its dual lamination.

The limit set $\Omega$ is the subset of $\bar{T}$ which consists of points with at least two pre-images by the map $\mathcal{Q}$. 
We also describe the trees such that $\operatorname{ind}_{\mathcal{Q}}(T)=2 N-2$ : these are the trees such that all points of $T$ have at least two pre-images by $\mathcal{Q}$.

Theorem 5.10. Let $T$ be an $\mathbb{R}$-tree in the boundary of Outer Space with dense orbits. The $Q$-index is maximal: $\operatorname{ind}_{\mathcal{Q}}(T)=2 N-2$ if and only if $T$ is contained in the limit set $\Omega$.

An $\mathbb{R}$-tree dual to a foliation on a surface with boundary of negative Euler characteristic, has maximal $\mathcal{Q}$-index. We call trees with maximal $\mathcal{Q}$-index trees of surface type.

1.4. Compact systems of isometries. A traditional strategy to study a tree in the boundary of Outer Space is

(1) describe any geometric tree by a system of isometries on a finite tree (or even a multi-interval) and then use the Rips machine;

(2) approximate any tree by a sequence of geometric trees.

In particular Gaboriau and Levitt [GL95] proved in this way that the geometric index of any tree in Outer Space is bounded above by $2 N-2$.

In [CHL09] a new approach was proposed: to describe an $\mathbb{R}$-tree by a system of isometries on a compact $\mathbb{R}$-tree (rather than on a finite tree). The point here is that any tree $T$ in the compactification of Outer Space can be described by a system of isometries on a compact $\mathbb{R}$-tree: $S_{A}=\left(K_{A}, A\right)$ (where $A$ is a basis of $\mathbb{F}_{N}$ and $K_{A}$ is a compact subtree of $\bar{T}$ ). This system of isometries encodes all of the original tree $T$ (together with the action of $\mathbb{F}_{N}$ ). An index is defined in Section 2.3 for any such system of isometries.

Theorem 5.7. Let $T$ be an $\mathbb{R}$-tree with a very small, minimal action of $\mathbb{F}_{N}$ by isometries with dense orbits. The Q $Q$-index of $T$ and the index of the induced system of isometries $S_{A}=\left(K_{A}, A\right)$, for any basis $A$, are equal:

$$
\operatorname{ind}_{\mathcal{Q}}(T)=i\left(S_{A}\right) .
$$

The computation of the index of a tree is thus achieved by computing the index of a system of isometries. We study system of isometries by themselves in Sections 2, 3 and 4.

We improve the classical Rips machine (see [GLP94], [BF95]) to work in the context of systems of isometries on compact $\mathbb{R}$-trees (or forests). The Rips machine applied to a system of isometries returns a new system of isometries obtained by erasing parts of the supporting forest. To each system of isometries we associate a finite graph $\Gamma$, the index of which is given by the Euler characteristic. We study the effect of the Rips machine on this graph: the Rips machine decreases the index of the graph $\Gamma$. 
Iterating the Rips machine infinitely many times, the sequence of associated graphs $\Gamma$ has a limit $\hat{\Gamma}$. The index of $\hat{\Gamma}$ is bounded above by the decreasing sequence of indices. We prove that the index of the limit graph $\widehat{\Gamma}$ is equal to the index of the system of isometries. In fact, in the case of a Levitt system of isometries the graph $\hat{\Gamma}$ can be viewed as a geometric realization of the dual lamination of the system of isometries. We obtain

Theorem 2.2. The index of a system of isometries $S=(F, A)$ with independent generators is finite and bounded above by the index of the associated graph $\Gamma$.

The above Theorem 5.10 follows from our characterization of systems of isometries with maximal index:

Theorem 4.8. Let $S=(F, A)$ be a reduced system of isometries with independent generators, let $\Gamma$ be its associated graph and $\widehat{\Gamma}$ be its limit graph. The following are equivalent:

(1) The system of isometries $S$ has maximal index.

(2) The graph $\hat{\Gamma}$ is finite.

(3) The Rips machine, starting from $S$, halts after finitely many steps.

Acknowledgment. We thank Martin Lustig for his constant interest in our work. We are grateful to Vincent Guirardel and Gilbert Levitt for introducing us to mixing properties of trees.

\section{Systems of isometries}

2.1. Definitions. We collect in this section basic facts from [CHL09].

An $\mathbb{R}$-tree, $(T, d)$ is a metric space such that for any two points $P, Q$ in $T$, there exists a unique arc $[P ; Q]$ between them and this arc is isometric to the segment $[0 ; d(P, Q)]$.

A compact forest $F$ is a metric space with finitely many connected components each of which is a compact $\mathbb{R}$-tree.

A partial isometry of a compact forest $F$ is an isometry $a: K \rightarrow K^{\prime}$, between two compact subtrees $K$ and $K^{\prime}$ of $F$. The domain of $a$ is $K$, its range is $K^{\prime}$. The partial isometry $a$ is non-empty if its domain is non-empty. The domain (and the range) of a partial isometry needs not be a whole connected component of $F$. A system of isometries $S=(F, A)$ consists of a compact forest $F$ and of a finite set $A$ of non-empty partial isometries of $F$.

To such a system of isometries $S$ we associate the oriented graph $\Gamma$ which has the connected components of $F$ as vertices and the non-empty partial isometries of $A$ as oriented edges. The edge $a \in A$ starts at the connected component of $F$ which 
contains its domain, and ends at the connected component of $F$ which contains its range.

We regard the reverse edge $a^{-1}$ of the edge $a \in A$ as the inverse partial isometry $a^{-1}$ of $a$. A reduced path $w$ in the graph $\Gamma$, given as a sequence of edges $w=z_{1} \ldots z_{n}$ with $z_{i} \in A^{ \pm 1}$ (such that $z_{i+1} \neq z_{i}{ }^{-1}$ ), defines a (possibly empty) partial isometry, also denoted by $w$ : the composition of partial isometries $z_{1} \circ z_{2} \circ \cdots \circ z_{n}$. We write this pseudo-action on $F$ on the right, i.e.

$$
P(u \circ v)=(P u) v
$$

for all points $P \in F$ and for all paths $u v$ in $\Gamma$.

The pseudo-orbit of a point $P$ in $F$ is the subset of $F$ which can be reached from $P$ :

$$
\{P \cdot w \mid w \text { reduced path in } \Gamma, P \in \operatorname{dom}(w)\} .
$$

A reduced path $w$ in $\Gamma$ is admissible if it is non-empty as a partial isometry of $F$.

An infinite reduced path $X$ in $\Gamma$ is admissible if all its subpaths are admissible. The domains of the initial subpaths of $X$ are nested compact subtrees, their intersection is the domain of $X$, denoted by $\operatorname{dom}(X)$.

A bi-infinite reduced path $Z$ in $\Gamma$ is admissible if all its subpaths are admissible. A bi-infinite reduced path, $Z=\ldots z_{-2} z_{-1} z_{0} z_{1} z_{2} \ldots$, has two halves which are infinite reduced paths:

$$
Z^{+}=z_{1} z_{2} \ldots, \quad Z^{-}=z_{0}^{-1} z_{-1}^{-1} z_{-2}^{-1} \ldots
$$

The domain of $Z$ is the intersection of the domains of its two halves. Equivalently, a bi-infinite reduced path $Z$ in $\Gamma$ is admissible if and only if its domain is non-empty.

The limit set $\Omega$ of a system of isometries $S=(F, A)$ is the set of elements of $K$ which are in the domain of a bi-infinite admissible reduced path in $\Gamma$. The limit set is the place where the dynamics of the system of isometries concentrates. Alternatively, $\Omega$ is the largest subset of $F$ such that for each $P \in \Omega$ there exists at least two partial isometries $a, b \in A^{ \pm 1}$ with $P \cdot a$ and $P \cdot b$ in $\Omega$.

A system of isometries $S=(F, A)$ has independent generators (compare Gaboriau [Gab97] and [CHL09]) if the domain of any admissible infinite reduced path $X$ in $\Gamma$ consists of exactly one point which we denote by $\mathcal{Q}(X)$ :

$$
\operatorname{dom}(X)=\{Q(X)\} .
$$

In this case, the domain of a bi-infinite admissible reduced path $Z$ in $\Gamma$ also consists of exactly one point which we also denote by $Q(Z)$.

If $S$ has independent generators we have

$$
\Omega=\{P \in K \mid \mathcal{Q}(Z)=P \text { for some bi-infinite admissible } \mathrm{Z}\} .
$$

The restriction of a partial isometry $a$ to the compact $\mathbb{R}$-tree $K$ (at the source) is the (possibly empty) partial isometry ${ }_{K\rceil} a$ which is defined for each $P \in K \cap \operatorname{dom}(a)$. The 
restriction of $a$ to the compact $\mathbb{R}$-tree $K^{\prime}$ (at the destination) is the (possibly empty) partial isometry $a_{\Gamma K^{\prime}}$ which is defined for each $P \in \operatorname{dom}(a)$ such that $P a \in K^{\prime}$. The restriction of the partial isometry to the compact $\mathbb{R}$-trees $K$ and $K^{\prime}$ is the (possibly empty) partial isometry ${ }_{K\rceil} a_{\left\lceil K^{\prime}\right.}$ which is defined for each $P \in K \cap \operatorname{dom}(a)$ such that $P \cdot a$ is in $K^{\prime}$.

2.2. Index of a graph. We denote by $V(\Gamma)$ the set of vertices of a graph $\Gamma$ and by $E(\Gamma)$ its set of edges.

For a vertex $x$ of a graph $\Gamma$ the valence $v_{\Gamma}(x)$ of $x$ is the number of edges incident to $x$. The index $i_{\Gamma}(x)=v_{\Gamma}(x)-2$ of $x$ is its valence minus two.

The index $i(\Gamma)$ of a finite connected graph $\Gamma$ is

$$
\begin{aligned}
i(\Gamma) & =\max \left(0 ; \sum_{x \in V(\Gamma)} i_{\Gamma}(x)\right) \\
& =\max (0 ; 2(\# E(\Gamma)-\# V(\Gamma))) \\
& =\max (0 ;-2 \chi(\Gamma)) \\
& =\max \left(0 ; 2 \operatorname{rank}\left(\pi_{1}(\Gamma)\right)-2\right),
\end{aligned}
$$

where $\chi(\Gamma)$ is the Euler characteristic of $\Gamma$. The index $i(\Gamma)$ is a homotopy invariant of the graph $\Gamma$.



Figure 3. A graph of index 2, with vertices of indices $i\left(v_{1}\right)=2, i\left(v_{2}\right)=0, i\left(v_{3}\right)=1$ and $i\left(v_{4}\right)=-1$.

The index of a finite graph $\Gamma$ is the sum of the indices of its connected components.

The core of a graph $\Gamma$ is the largest subgraph of $\Gamma$ without vertices of valence 0 and 1 , we denote it by core $(\Gamma)$. The core of a graph is the union of all bi-infinite reduced paths. Alternatively, if $\Gamma$ is finite, the subgraph core $(\Gamma)$ is obtained by recursively erasing vertices of valence 0 and the vertices of valence 1 together with their incident edges. The core of a graph may be empty: this is the case, for instance, when the graph is a union finite of trees.

Our use of core is not that of Stallings [Sta83] (except in the case of finite graph), but it agrees with the notion of normalization of Gersten [Ger83].

The index of a finite graph can be computed using its core with the easier formula:

$$
i(\Gamma)=i(\operatorname{core}(\Gamma))=\sum_{x \in V(\operatorname{core}(\Gamma))} i_{\operatorname{core}(\Gamma)}(x) .
$$


We use this formula to define the index of an infinite graph $\Gamma$ : The core does not have vertices of valence 0 and 1 and thus we can compute the above (possibly infinite) non-negative sum.

For a connected (possibly infinite) graph $\Gamma$ the index is thus

$$
i(\Gamma)=i(\operatorname{core}(\Gamma))=\max \left(0 ; \# \partial \Gamma+2 \operatorname{rank}\left(\pi_{1}(\Gamma)\right)-2\right)
$$

where $\partial \Gamma$ is the set of ends of $\Gamma$. If $\Gamma$ is not connected we sum the above value for each of its connected components.

A morphism of graphs $\tau: \Gamma^{\prime} \rightarrow \Gamma$ maps vertices to vertices, edges to edges and respects incidence.

We will need the following lemma in our proofs:

Lemma 2.1. Let $\tau: \Gamma^{\prime} \rightarrow \Gamma$ be a morphism between two finite graphs. Assume that $\tau$ is injective on edges. Then the index of $\Gamma^{\prime}$ is smaller or equal to the index of $\Gamma$ :

$$
i\left(\Gamma^{\prime}\right) \leq i(\Gamma)
$$

Proof. For each vertex $x$ of $\Gamma$, the set of edges incident to the vertices in the fiber $\tau^{-1}(x)$ injects in the set of edges incident to $x$. Thus

$$
\sum_{x^{\prime} \in \tau^{-1}(x)} i_{\Gamma^{\prime}}\left(x^{\prime}\right) \leq i_{\Gamma}(x)
$$

Moreover, $\tau$ maps the core of $\Gamma^{\prime}$ inside the core of $\Gamma$.

We get

$$
\begin{aligned}
i\left(\Gamma^{\prime}\right) & =i\left(\operatorname{core}\left(\Gamma^{\prime}\right)\right)=\sum_{x \in V\left(\operatorname{core}\left(\Gamma^{\prime}\right)\right)} i_{\operatorname{core}\left(\Gamma^{\prime}\right)}(x) \leq \sum_{x \in V(\operatorname{core}(\Gamma))} i_{\operatorname{core}(\Gamma)}(x) \\
& =i(\operatorname{core}(\Gamma))=i(\Gamma)
\end{aligned}
$$

In particular, if $\Gamma^{\prime}$ is a subgraph of a finite graph $\Gamma$,

$$
i\left(\Gamma^{\prime}\right) \leq i(\Gamma)
$$

2.3. Index of a system of isometries. Let $F$ be a compact forest and $S=(F, A)$ be a system of isometries. Let $\Omega$ be the limit set of $S$.

For a point $P$ in $F$, we define its index by

$$
i_{S}(P)=\#\left\{a \in A^{ \pm 1} \mid P \cdot a \in \Omega\right\}-2 .
$$

By definition of the limit set, for any point $P$ in $\Omega$, there exists a bi-infinite reduced admissible path $Z=\ldots z_{-1} z_{0} z_{1} \ldots$ in $\Gamma$ such that $P \in \operatorname{dom}(Z)$. The edges $z_{1}$ and $z_{0}{ }^{-1}$ send $P$ inside $\Omega$, and thus the index of $P$ is greater or equal to $0: i_{S}(P) \geq 0$. 
Conversely, if the index of a point $P$ in $F$ is non-negative: $i_{S}(P) \geq 0$, then there exists two elements $a, b \in A^{ \pm 1}$, such that $P \cdot a$ and $P \cdot b$ are in the limit set $\Omega$. As $P \cdot a$ is in $\Omega$ there exists a bi-infinite reduced admissible path $Z=\ldots z_{-1} z_{0} z_{1} \ldots$ in $\Gamma$ such that $P \cdot a \in \operatorname{dom}(Z)$. Up to replacing $Z$ by $Z^{-1}$ (the same bi-infinite path with reversed orientation), we assume that $z_{1} \neq a^{-1}$. Symmetrically there exists a biinfinite reduced admissible path $Z^{\prime}=\ldots z_{-1}^{\prime} z_{0}^{\prime} z_{1}^{\prime} \ldots$ in $\Gamma$ such that $P \cdot b \in \operatorname{dom}\left(Z^{\prime}\right)$ and $z_{0}^{\prime} \neq b^{-1}$. We get that $Z^{\prime \prime}=\ldots z_{-1}^{\prime} z_{0}^{\prime} b \dot{a} z_{1} z_{2} \ldots$ is a bi-infinite reduced path in $\Gamma$ with $P \in \operatorname{dom}\left(Z^{\prime \prime}\right)$ and thus that $P$ is in the limit set $\Omega$ :

$$
P \in \Omega \Longleftrightarrow i_{S}(P) \geq 0
$$

The index of $S$ is defined by

$$
i(S)=\sum_{P \in F} \max \left(0 ; i_{S}(P)\right)=\sum_{P \in \Omega} i_{S}(P) .
$$

As there is a max in the first sum, and by the above equivalence, both sums are non-negative and are well defined possibly as $+\infty$.

The main result of this paper can now be stated.

Theorem 2.2. The index of a system of isometries $S=(F, A)$ with independent generators is finite and bounded above by the index of the associated graph $\Gamma$.

2.4. Cayley graphs. Let $S=(F, A)$ be a system of isometries and let $\Gamma$ be its associated graph. Let $P$ be a point in $F$ and $K$ be the connected component of $F$ which contains $P$. Let $\Gamma_{0}$ be the connected component of $\Gamma$ which contains $K$ and $\tilde{\Gamma}_{0}$ its universal cover.

The trajectory tree of $P$ is the smallest subtree $\widetilde{\Gamma}(P)=\widetilde{\Gamma}(P, S)$ of $\tilde{\Gamma}_{0}$ which contains all the admissible paths $w$ based at $K$ such that $P$ is in the domain of $w$.

Let $\operatorname{Stab}(P)$ be the subgroup of the fundamental group $\pi_{1}(\Gamma, K)$ of the graph $\Gamma$ based at $K$ of admissible paths $w$ such that $P \cdot w=P$. The group $\operatorname{Stab}(P)$ is a free group that acts on the tree $\widetilde{\Gamma}(P)$.

The Cayley graph $\Gamma(P)=\Gamma(P, S)$ of $P$ is the quotient of $\widetilde{\Gamma}(P)$ by the action of the stabilizer $\operatorname{Stab}(P)$ (compare Gaboriau [Gab96]). Vertices of the Cayley graph of $P$ are in one-to-one correspondence with the elements of the pseudo-orbit of $P$ in $F$ under the pseudo-group of isometries. The vertices of the core of the Cayley graph of $P$ are in one-to-one correspondence with the intersection $\omega(P)$ of the pseudo-orbit of $P$ and the limit set $\Omega$.

The index of a vertex $P^{\prime}$ in $\operatorname{core}(\Gamma(P))$ is equal to the index $i_{S}\left(P^{\prime}\right)$ of the point $P^{\prime}$ for the system of isometries $S$. Thus, we get that the index of the core of the Cayley graph $i(\Gamma(P))$ is equal to the contribution of the pseudo-orbit of $P$ to the index of $S$ :

$$
i(\Gamma(P))=i(\operatorname{core}(\Gamma(P)))=\sum_{P^{\prime} \in \omega(P)} i_{S}\left(P^{\prime}\right)
$$


Adding up, for all pseudo-orbits $[P]$ we get

$$
i(S)=\sum_{[P]} i(\Gamma(P)) .
$$

\section{Rips machine}

3.1. Elementary step. Let $S=(F, A)$ be a system of isometries on a compact forest $F$. One step of the Rips machine produces a new system of isometries $S^{\prime}=\left(F^{\prime}, A^{\prime}\right)$ defined as follows.

The forest $F^{\prime}$ is the set of all elements of $F$ which are in the domains of at least two distinct partial isometries in $A^{ \pm 1}$ :

$$
F^{\prime}=\left\{P \in F \mid \text { there exists } a \neq b \in A^{ \pm 1} \text { such that } P \in \operatorname{dom}(a) \cap \operatorname{dom}(b)\right\} .
$$

The set $F^{\prime}$ has finitely many connected components which are compact $\mathbb{R}$-trees because it is the finite union of all possible intersections $\operatorname{dom}(a) \cap \operatorname{dom}(b)$ for all pairs of distinct elements $a, b$ of $A^{ \pm 1}$.

For each partial isometry $a \in A$ and for each pair of connected components $K_{0}, K_{1}$ of $F^{\prime}$, we consider the partial isometry, $a^{\prime}={ }_{\left.K_{0}\right\rceil} a_{\left\lceil K_{1}\right.}$, which is the restriction of $a$ to $K_{0}$ and $K_{1}$. The finite set $A^{\prime}$ consists of all such non-empty partial isometries $\left.K_{0}\right\rceil_{\left\lceil K_{1}\right.}$ of $F^{\prime}$.

An elementary step of the Rips machine gives rise to a map $\tau$ from the graph $\Gamma^{\prime}$ associated to the resulting system of isometries $S^{\prime}$, to the original graph $\Gamma$. A vertex $K^{\prime}$ of $\Gamma^{\prime}$ is a connected component of $F^{\prime}$ and it is mapped by $\tau$ to the connected component $\tau\left(K^{\prime}\right)$ of $F$ which contains $K^{\prime}$. Similarly an edge $a^{\prime}$ of $\Gamma^{\prime}$ is a non-empty partial isometry $a^{\prime}=K_{0} a_{\left\lceil K_{1}\right.}$ and it is mapped by $\tau$ to the original partial isometry $a$. The map $\tau$ is a morphism of oriented graphs.

If $w$ is an admissible reduced path in $\Gamma^{\prime}$, the domain of $\tau(w)$ contains the domain of $w$ and $\tau(w)$ is an admissible path in $\Gamma$. Moreover, the image $\tau(w)$ of an admissible reduced path $w$ in $\Gamma^{\prime}$ is a reduced path of $\Gamma$. Finally, if a bi-infinite reduced path $Z$ in $\Gamma$ is admissible, then its domain is contained in $F^{\prime}$, which leads to the following

Proposition 3.1. Let $S$ be a system of isometries and $S^{\prime}$ be the result of the Rips machine. Then the limit sets and the indices of $S$ and $S^{\prime}$ are equal:

$$
\Omega=\Omega^{\prime} \quad \text { and } \quad i(S)=i\left(S^{\prime}\right) .
$$

3.2. Indices through the Rips machine. As explained previously, the Rips machine defines a new system of isometries $S^{\prime}=\left(F^{\prime}, A^{\prime}\right)$ starting from a system of isometries $S=(F, A)$ by erasing the subset $E$ of the forest $F$ which consists of points which belongs to at most one domain of partial isometries of $A^{ \pm 1}$.

To better understand the Rips machine we decompose its elementary step into a finite sequence of elementary moves. Instead of erasing $E$ in one step we successively 
erase subsets $E_{i}$ of $E$. This gives us a finite sequence of system of isometries starting from $S$ and ending at $S^{\prime}$. The successive systems of isometries of this sequence differ by an elementary move.

As $F$ is a compact forest, the set $E$ may have infinitely many connected components. We first describe a preliminary move which erases all the "peripheral" ones. Then we are left with a finite forest to erase, which we erase in finitely many elementary moves.

This decomposition of the Rips machine is used in the next proposition to prove that the index of the associated graphs is decreasing.

Proposition 3.2. Let $S=(F, A)$ be a system of isometries and $S^{\prime}=\left(F^{\prime}, A^{\prime}\right)$ be the output of the Rips machine. Let $\Gamma$ and $\Gamma^{\prime}$ be the associated graphs.

Then the index $i\left(\Gamma^{\prime}\right)$ is smaller or equal than the index $i(\Gamma)$.

Proof. Let $E$ be the part of the forest $F$ which is erased by the Rips machine:

$$
E=F \backslash F^{\prime}=\left\{P \in F \mid \#\left\{a \in A^{ \pm 1} \mid P \in \operatorname{dom}(a)\right\} \leq 1\right\} .
$$

Let $E_{C}$ be the subset of $E$ which is in the convex hull of $F^{\prime}$ :

$$
E_{C}=\left\{P \in E \mid P \in[Q, R] \text { for some } Q, R \in F^{\prime}\right\},
$$

and let $E_{0}=E \backslash E_{C}$ be the complement of $E_{C}$ in $E$.

Let $F_{0}=F \backslash E_{0}=F^{\prime} \cup E_{C}: F_{0}$ is the convex hull of the connected components of $F^{\prime}$ in $F$ :

$$
F_{0}=\left\{P \in F \mid P \in[Q, R] \text { for some } Q, R \in F^{\prime}\right\} .
$$

Thus $F_{0}$ has finitely many connected components, each of which is a compact $\mathbb{R}$-tree: $F_{0}$ is a compact forest.

Let $A_{0}$ be the set of all non-empty restrictions of partial isometries of $A$ to partial isometries of $F_{0}$. Let $\Gamma_{0}$ be the graph associated to the system of isometries $S_{0}=$ $\left(F_{0}, A_{0}\right)$ and let $\tau_{0}: \Gamma_{0} \rightarrow \Gamma$ be the graph morphism defined as before. As $E_{0}$ does not split connected components of $F$, the map $\tau_{0}$ is injective and thus by Lemma 2.1,

$$
i\left(\Gamma_{0}\right) \leq i(\Gamma)
$$

(we note that this inequality can be strict if $\tau_{0}$ is not onto).

As $F^{\prime}$ has finitely many connected components, $E_{C}$ is a finite union of finite open arcs of the form ] $P ; Q$ [ where $P$ and $Q$ are two points of $F^{\prime}$.

Lemma 3.3. The erased part $E_{C}$ can be decomposed to get a partition

$$
E_{C}=\alpha_{1} \uplus \alpha_{2} \uplus \cdots \uplus \alpha_{n}
$$

where each $\alpha_{i}$ is an open arc such that the number of connected components of $F_{i}$ is exactly one plus the number connected components of $F_{i-1}$, where for each $i=1, \ldots, n$, we let $F_{i}=F_{i-1} \backslash \alpha_{i}$. 
Proof. We recursively define $\alpha_{i}$ by choosing a connected component $C$ of $F_{i-1}$ which contains at least two connected components of $F^{\prime}$. Then we choose a connected component $K$ of $F^{\prime}$ contained in $C$ and which is not contained in the convex hull $C^{\prime}$ of $\left(C \cap F^{\prime}\right) \backslash K$ in $C$. We choose the open arc $\alpha_{i}$ that joins $K$ and $C^{\prime}$. Removing $\alpha_{i}$ from $F_{i-1}$ splits the connected component $C$ of $F_{i-1}$ into two new connected components: $K$ and $C^{\prime}$.

Let $A_{i}$ be the set of non-empty restrictions of partial isometries in $A$ to $F_{i}$ and let $\Gamma_{i}$ be the graph associated to the system of isometries $S_{i}=\left(F_{i}, A_{i}\right)$. As before we get graph morphisms $\tau_{i}: \Gamma_{i} \rightarrow \Gamma_{i-1}$. We observe that the last system of isometries is the output of the Rips machine: $S_{n}=S^{\prime}$. The map $\tau$ factors through the graphs $\Gamma_{i}: \tau=\tau_{0} \circ \tau_{1} \circ \cdots \circ \tau_{n}$.

We now proceed to prove that for each $i=1, \ldots, n$, the index of $\Gamma_{i}$ is lower or equal to the index of $\Gamma_{i-1}$. This will conclude the proof.

Going from $S_{i-1}$ to $S_{i}$ corresponds to one of the following elementary moves of the Rips machine. For each $i$ removing the $\operatorname{arc} \alpha_{i}$ from $F_{i-1}$ has one of the following two effects on the graph $\Gamma_{i-1}$ :

(1) Split a vertex: $\alpha_{i}$ joins two connected components, $K$ and $K^{\prime}$, of $F_{i}$, and no partial isometry in $A_{i-1}^{ \pm 1}$ is defined simultaneously on $K$ and $K^{\prime}$. Then the map $\tau_{i}$ is injective on edges and, applying Lemma 2.1, the index of $\Gamma_{i}$ is smaller or equal to the index of $\Gamma_{i-1}$.



Figure 4. Split a vertex move.

(2) Split an edge: $\alpha_{i}$ joins two connected components $K$ and $K^{\prime}$ of $F_{i}$, and a partial isometry $a$ in $A_{i-1}^{ \pm 1}$ is defined on $K$ and $K^{\prime}$ (and thus its domain $\operatorname{dom}(a)$ contains $\alpha_{i}$ ). By definition of $E$ no other partial isometry is defined on $\alpha_{i}$, in particular the range of $a$ is contained in $F_{i}$. The graph morphism $\tau_{i}$ maps the two distinct vertices $K$ and $K^{\prime}$ to the same vertex, $K \cup \alpha_{i} \cup K^{\prime}$, of $\Gamma_{i-1}$, it maps the two edges $a^{\prime}={ }_{K\rceil} a$ and $a^{\prime \prime}={ }_{K^{\prime}} a$ which are the restrictions of $a$ to $K$ and $K^{\prime}$ to the same edge $a$ of $\Gamma_{i-1}$. 

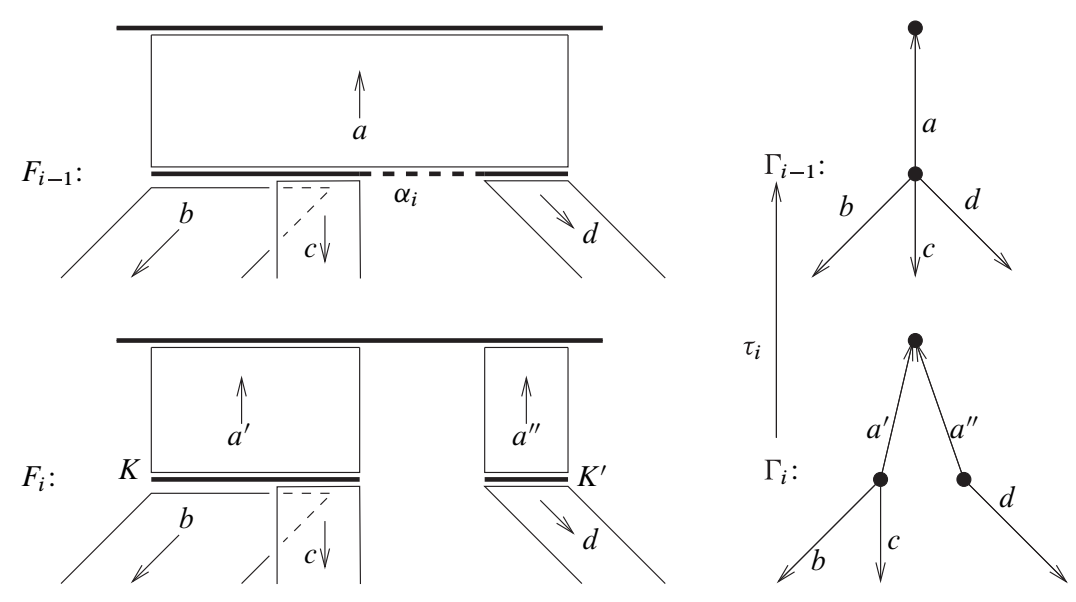

Figure 5. Split an edge move.

On all other vertices and edges, $\tau_{i}$ is one-to-one. Thus, $\tau_{i}$ is a homotopy equivalence and the index of $\Gamma_{i}$ is equal to the index of $\Gamma_{i-1}$. Indeed, $\tau_{i}$ is a folding in Stallings' terminology [Sta83].

3.3. Iterating the Rips machine. Let $S_{0}=\left(F_{0}, A_{0}\right)$ be a system of isometries. By repeatedly applying the Rips machine we get a sequence $S_{n}=\left(F_{n}, A_{n}\right)$ of systems of isometries. Of course the Rips machine may halt after some time, that is to say, we do not exclude that $S_{n+1}=S_{n}$ for $n$ big enough.

We also get graph morphisms $\tau_{n}$ from the graph $\Gamma_{n+1}$ associated to $S_{n+1}$ to the graph $\Gamma_{n}$ associated to $S_{n}$. Indeed, a partial isometry $a_{n} \in A_{n}$ is the restriction of the partial isometry $a=\tau_{0} \circ \tau_{1} \cdots \tau_{n-1}\left(a_{n}\right)$ in $A_{0}$ to connected components of the compact forest $F_{n}$.

Lemma 3.4. The intersection $\Omega$ of the nested sequence $\left(F_{n}\right)_{n \in \mathbb{N}}$ of compact subsets of $F_{0}$ is equal to the limit set $\Omega_{0}$ of the system of isometries $S_{0}$.

Proof. By Proposition 3.1, at each step $n$ the limit sets $\Omega_{n+1}$ and $\Omega_{n}$ of the corresponding system of isometries are equal. In particular $\Omega_{0}$ is contained in $F_{n}$ at each step $n$ and thus in the nested intersection.

Conversely, let $P$ be a point in the nested intersection $\Omega=\bigcap_{n \in \mathbb{N}} F_{n}$. For any $n \in \mathbb{N}, P$ belongs to $F_{n+1}$ and by definition of the Rips machine, there exists at least two distinct partial isometries $a_{n}$ and $b_{n}$ defined at $P$ in ${A_{n}}^{ \pm 1}$. Thus $P \cdot a_{n}$ and $P \cdot b_{n}$ are in $F_{n}$. Up to passing to a subsequence we can assume that $a_{n}$ and $b_{n}$ are the restrictions of two fixed and distinct partial isometries $a$ and $b$ in $A_{0}^{ \pm 1}$. We get that for all $n, P \cdot a$ and $P \cdot b$ are in $F_{n}$, which proves that $P \cdot a$ and $P \cdot b$ are also in the nested intersection $\Omega=\bigcap_{n \in \mathbb{N}} F_{n}$. The set $\Omega$ is a subset of $F_{0}$ such that for any 
point $P$ in $\Omega$, there exists at least two distinct partial isometries $a$ and $b$ in $A_{0}^{ \pm 1}$ such that $P \cdot a$ and $P \cdot b$ are also in $\Omega$. This proves that $\Omega$ is contained in the limit set $\Omega_{0}$ of the initial system of isometries $S_{0}$.

The limit graph $\hat{\Gamma}$ of the system of isometries $S_{0}=\left(F_{0}, A_{0}\right)$ is the (possibly infinite) graph whose vertices are the connected components of the limit set $\Omega$ and whose edges are all possible restrictions of partial isometries in $A_{0}$ to connected components of $\Omega$. We denote by $\hat{\tau}_{n}: \hat{\Gamma} \rightarrow \Gamma_{n}$ the graph morphism that maps a connected component $C$ of $\Omega$ to the connected component of $F_{n}$ that contains $C$, and which maps an edge $e$ of $\widehat{\Gamma}$ to the partial isometry $a_{n} \in A_{n}$ of which it is a restriction.

From the previous lemma and from the definition of an inverse limit, we deduce:

Lemma 3.5. The limit graph $\hat{\Gamma}$ of a system of isometries, $S_{0}=\left(F_{0}, A_{0}\right)$, is the inverse limit of the sequence of graphs $\left(\Gamma_{n}\right)_{n \in \mathbb{N}}$ (together with the sequence of maps $\left.\left(\tau_{n}\right)_{n \in \mathbb{N}}\right)$ associated to the sequence of systems of isometries $\left(S_{n}\right)_{n \in \mathbb{N}}$ obtained from $S_{0}$ by iterating the Rips machine.

By definition of $\Omega$, the graph $\widehat{\Gamma}$ does not have vertices of valence 0 or 1 , and we defined its index as the non-negative sum

$$
i(\widehat{\Gamma})=\sum_{x \in V(\widehat{\Gamma})} i_{\widehat{\Gamma}}(x)
$$

Recall that, by Proposition 3.2, the sequence of indices $\left(i\left(\Gamma_{n}\right)\right)_{n \in \mathbb{N}}$ is decreasing.

Proposition 3.6. Let $\widehat{\Gamma}$ be the limit graph of a system of isometries $S_{0}=\left(F_{0}, A_{0}\right)$. Then the index of $\widehat{\Gamma}$ is smaller or equal to the index of $\Gamma_{0}$ :

$$
i(\widehat{\Gamma}) \leq i\left(\Gamma_{0}\right)
$$

Proof. For any point $P$ in $\Omega$, by definition, there exist at least two distinct partial isometries $a$ and $b$ in $A_{0}^{ \pm 1}$ defined at $P$ and such that $P \cdot a$ and $P \cdot b$ also lie in $\Omega$. For any $n \in \mathbb{N}, \Omega$ is contained in $F_{n}$ : let $C_{n}$ be the connected component of $P$ in $F_{n}$. There are at least two edges going out of the vertex $C_{n}$ of $\Gamma_{n}$ corresponding to the restrictions of $a$ and $b$ to $C_{n}$. This proves that the image of $\widehat{\Gamma}$ by $\hat{\tau}_{n}$ in $\Gamma_{n}$ does not contain vertices of valence 0 or $1: \hat{\tau}_{n}(\hat{\Gamma})$ is a subgraph of the core of $\Gamma_{n}$.

Let $\Theta_{0}$ be a finite set of vertices of $\widehat{\Gamma}$. Let $\Theta$ be a finite subgraph of $\widehat{\Gamma}$ that contains $\Theta_{0}$ and all edges incident to elements of $\Theta_{0}$. The graph $\Theta$ exists because vertices of $\widehat{\Gamma}$ have finite valence bounded above by twice the cardinality of $A_{0}$.

By Lemma 3.5, there exists $n \in \mathbb{N}$ such that $\Theta$ is mapped injectively by $\hat{\tau}_{n}$ into $\Gamma_{n}$. Arguing as in Lemma 2.1 and using the definition of the index of a graph, the following inequalities hold:

$$
\sum_{x \in \Theta_{0}} i_{\widehat{\Gamma}}(x)=\sum_{x \in \Theta_{0}} i_{\Theta}(x) \leq \sum_{x \in \Theta_{0}} i_{\operatorname{core}\left(\Gamma_{n}\right)}\left(\hat{\tau}_{n}(x)\right) \leq i\left(\operatorname{core}\left(\Gamma_{n}\right)\right)=i\left(\Gamma_{n}\right) .
$$


Using Proposition 3.2 we get that for any finite subset $\Theta_{0}$ of vertices of $\widehat{\Gamma}$

$$
\sum_{x \in \Theta_{0}} i_{\widehat{\Gamma}}(x) \leq i\left(\Gamma_{0}\right) .
$$

Taking $\Theta_{0}$ arbitrarily large we finally get

$$
i(\widehat{\Gamma}) \leq i\left(\Gamma_{0}\right)
$$

A connected component $K$ of $\Omega$ is called regular if it corresponds to a vertex of valence 2 of the limit graph $\widehat{\Gamma}$. A connected component $K$ of $\Omega$ is singular if it corresponds to a vertex of valence at least 3 of $\hat{\Gamma}$.

Corollary 3.7. All connected components of $\widehat{\Gamma}$ are lines except at most $i\left(\Gamma_{0}\right)$. Moreover, there are at most $i\left(\Gamma_{0}\right)$ singular connected components of $\Omega$.

We are now ready to prove Theorem 2.2 in the special case where the Rips machine never halts and digs holes everywhere.

Theorem 3.8. Let $S=(F, A)$ be system of isometries and $\Gamma$ be its associated graph. Assume that the limit set $\Omega$ is totally disconnected. Then the index of $S$ is bounded above by the index of $\Gamma$.

Proof. As connected components of $\Omega$ are reduced to single points, the graph $\hat{\Gamma}$ is the disjoint union of all the cores of the Cayley graphs $\Gamma(P)$ :

$$
\widehat{\Gamma}=\biguplus_{[P]} \operatorname{core}(\Gamma(P)) .
$$

Thus, the index of $\widehat{\Gamma}$ is equal to the index of $S$. The theorem now follows from Proposition 3.6.

We turn back to the general case where the limit set has non-trivial (i.e. not reduced to a single point) connected components.

Proposition 3.9. The limit set $\Omega$ of a system of isometries $S_{0}=\left(F_{0}, A_{0}\right)$ has finitely many non-trivial connected components.

Proof. By Corollary 3.7, there are finitely many singular connected components of $\Omega$.

Let $K$ be a regular connected component of $\Omega$. Then there exists exactly two distinct partial isometries $a \neq b$ in $A_{0}{ }^{ \pm 1}$ with non trivial restrictions $a^{\prime}={ }_{K\rceil} a_{\lceil\Omega}$ and $b^{\prime}={ }_{K\rceil} b_{\lceil\Omega}$ to $K$ and $\Omega$. These are the partial isometries which give rise to the two edges of $\widehat{\Gamma}$ going out of the vertex $K$. By definition of $\Omega$, for each point $P$ of $K$ there exists at least two partial isometries in $A_{0}^{ \pm 1}$ which map $P$ inside $\Omega$. Thus $P$ lies in both the domains of $a^{\prime}$ and $b^{\prime}$ and thus $\operatorname{dom}\left(a^{\prime}\right)=\operatorname{dom}\left(b^{\prime}\right)=K$. 
Now, if the range $K \cdot a^{\prime}$ of $a^{\prime}$ is also a regular connected component of $\Omega$ then $a^{\prime}$ is an isometry between $K$ and $K \cdot a^{\prime}$ and in particular they have the same diameter. From the next lemma we get that there can only be finitely many non-trivial regular connected components.

This proves that $\Omega$ only has finitely many non-trivial connected components.

Lemma 3.10. Let $K$ be a compact $\mathbb{R}$-tree and $\left(K_{i}\right)_{i \in \mathbb{N}}$ be a collection of disjoint subtrees of $K$. Then

$$
\lim _{i \rightarrow \infty} \operatorname{diam}\left(K_{i}\right)=0 .
$$

Proof. By contradiction, assume that there exists $\varepsilon>0$ such that (extracting a subsequence) for all $i, \operatorname{diam}\left(K_{i}\right)>\varepsilon$. Let $P_{i}, Q_{i}$ be in $K_{i}$ with $d\left(P_{i}, Q_{i}\right) \geq \varepsilon$. By compactness of $K$, up to passing to a subsequence, the sequences $P_{i}$ and $Q_{i}$ converge to $P$ and $Q$ respectively in $K$ and $d(P, Q) \geq \varepsilon$. For $i$ big enough $d\left(P_{i}, P\right)<\varepsilon / 2$ and $d\left(Q_{i}, Q\right)<\varepsilon / 2$, from which we deduce that the segment $\left[P_{i}, Q_{i}\right]$ contains the midpoint of $[P, Q]$, and therefore the segments $\left[P_{i}, Q_{i}\right]$ and $\left[P_{j}, Q_{j}\right]$ are not disjoint for $i$ and $j$ big enough. A contradiction.

3.4. Reduced systems of isometries. In this section we introduce an extra hypothesis on the system of isometries. Under this hypothesis using the Rips machine is considerably easier. For system of isometries on finite trees, such an extra hypothesis was introduced by D. Gaboriau in [Gab96] where it appears in Proposition V.4 as Property (*).

The set of extremal points $\partial K$ of a compact $\mathbb{R}$-tree $K$ is the set of points of $K$ that do not lie in the interior of an interval contained in $K$. Equivalently a point $P$ is extremal in $K$ if $K \backslash\{P\}$ is connected. The tree $K$ is the convex hull of its extremal points:

$$
K=\bigcup_{P, P^{\prime} \in \partial K}\left[P ; P^{\prime}\right]
$$

We remark that $\partial K$ may fail to be compact.

Definition 3.11. Let $S_{0}=\left(F_{0}, A_{0}\right)$ be a system of isometries. Let $S_{1}=\left(F_{1}, A_{1}\right)$ be the output of the Rips machine. The system of isometries $S_{0}$ is reduced if

(i) For any point $P$ in $F_{0}$ the tree of trajectories $\widetilde{\Gamma}(P)$ is infinite.

(ii) For any partial isometry $a$ in $A_{0}^{ \pm 1}$ the set of extremal points $\partial \operatorname{dom}(a)$ of the domain of $a$ is contained in $F_{1}$.

Lemma 3.12. The graph $\Gamma_{0}$ associated to a reduced system of isometries does not have vertices of negative index, that is to say of valence 0 or 1 .

Proof. Let $K$ be a connected component of $F$ and let $P$ be a point in $K$. From condition (i), the tree of trajectories $\widetilde{\Gamma}(P)$ is infinite and thus there exists at least one 
partial isometry $a \in A_{0}^{ \pm 1}$ defined at $P$. Let now $Q$ be an extremal point of $\operatorname{dom}(a)$. From condition (ii), $Q$ is in $F_{1}$ and thus belongs to at least another domain $\operatorname{dom}(b)$ with $b \in A_{0}^{ \pm 1}, a \neq b$. Thus, the vertex $K$ of $\Gamma_{0}$ has at least two outgoing edges $a$ and $b$.

When dealing with the Rips machine, reduced systems of isometries are easier to handle. The elementary moves "split a vertex" and "split an edge" are described in the proof of Proposition 3.2.

Proposition 3.13. Let $S_{0}=\left(F_{0}, A_{0}\right)$ be a reduced system of isometries. Let $S_{1}=$ $\left(F_{1}, A_{1}\right)$ be the output of the Rips machine.

Then going from $S_{0}$ to $S_{1}$ only performs elementary moves of type "split an edge", and the map $\tau: \Gamma_{1} \rightarrow \Gamma_{0}$ is a homotopy equivalence. In particular

$$
i\left(\Gamma_{0}\right)=i\left(\Gamma_{1}\right) .
$$

Proof. In the proof of Proposition 3.2, starting with a reduced system of isometries, we first get that $E_{0}$ is empty. Indeed, let $P$ be an extremal point of $F$ which is not in $F_{1}$. From condition (ii), $P$ does not belong to any domain of a partial isometry in $A^{ \pm 1}$ and thus its tree of trajectories consists in a single vertex, which contradicts condition (i).

Then we get that no "split a vertex" move can occur, because the removed points in this move have a tree of trajectories which consists in a single vertex.

We can now prove that the reduced condition is inherited while iterating the Rips machine.

Proposition 3.14. Let $S_{0}=\left(F_{0}, A_{0}\right)$ be a reduced system of isometries. Let $S_{1}=$ $\left(F_{1}, A_{1}\right)$ be the output of the Rips machine. Then $S_{1}$ is reduced.

Proof. For a point $P$ in $F_{1}$, the tree of trajectories of $P$ with respect to $S_{1}$ is obtained from the tree of trajectories with respect to $S_{0}$ by pruning off the terminal vertices. Thus, if the latter is infinite, so is the former.

By contradiction, let $S_{2}=\left(F_{2}, A_{2}\right)$ be the output of the Rips machine applied to $S_{1}$, let $a_{1}$ be a partial isometry in $A_{1}$ and let $P$ be an extremal point in $\partial \operatorname{dom}\left(a_{1}\right)$ which is not in $F_{2}$. Let $a_{0}$ be the partial isometry in $A_{0}$ of which $a_{1}$ is a restriction.

As $P$ is in $\operatorname{dom}\left(a_{1}\right)$, and thus in $F_{1}$, there is at least another partial isometry $b_{0} \in A_{0}$ such that $P$ is in the domain $\operatorname{dom}\left(b_{0}\right)$. There exist two extremal points $Q$, $R$ in $\partial \operatorname{dom}\left(b_{0}\right)$ such that $P$ lies in the segment $[Q ; R]$. By hypothesis, $Q, R$, as well as $Q \cdot b_{0}$ and $R \cdot b_{0}$ lie in $F_{1}$.

As $P$ is an extremal point in $\partial \operatorname{dom}\left(a_{1}\right)$, up to exchanging the two points $Q$ and $R$, we assume that $Q$ is not in the domain of $a_{1}$ and that $[P ; Q] \cap \operatorname{dom}\left(a_{1}\right)=\{P\}$. Let $\left(Q_{n}\right)$ be a sequence of points in the open arc ] $P ; Q[$ that converges to $P$. 


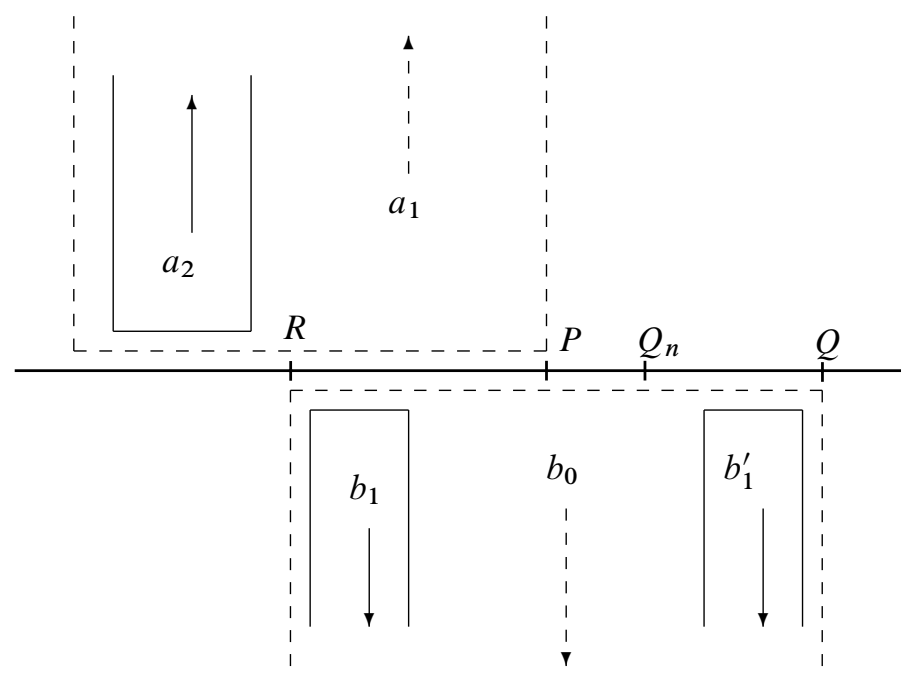

In the original system of isometries $S_{0}, Q_{n}$ has an infinite tree of trajectories, in particular there exists a reduced admissible path $c_{n} \cdot d_{n}$ of length 2 in $\Gamma_{0}$ which is defined at $Q_{n}$. As $\Gamma_{0}$ is a finite graph, up to passing to a subsequence we assume that this path is constant: for any $n$ the reduced admissible path $c \cdot d$ is defined at $Q_{n}$. As domains of partial isometries are closed, the point $P$ is in the domain of $c . d$. Since $c^{-1} \neq d$, by definition of the Rips machine, the point $P c$ is in $F_{1}$ and there is a restriction $c_{1}$ of $c$ in $A_{1}$ which is defined at $P$. As $P$ is not in $F_{2}$ and as $a_{1}$ is defined at $P$, the partial isometries $c_{1}$ and $a_{1}$ are equal. Thus for any integer $n$ the partial isometry $a_{0}$ is defined at $Q_{n}$ and $Q_{n} a_{0}$ is in $F_{1}$. There are only finitely many restrictions of $a_{0}$ to the connected components of $F_{1}$. Thus $a_{1}$ is defined at some $Q_{n}$ and $P$ is not an extremal point in $\partial \operatorname{dom}\left(a_{1}\right)$. A contradiction.

We now state an equivalent characterization of reduced systems of isometries.

Proposition 3.15. A system of isometries $S_{0}=\left(F_{0}, A_{0}\right)$ is reduced if and only if the two following conditions hold:

(i) For any point $P$ in $F$ the tree of trajectories $\widetilde{\Gamma}(P)$ is infinite.

(ii') For any partial isometry a in $A^{ \pm 1}$ the set of extremal points $\partial \operatorname{dom}(a)$ of the domain of a is contained in the limit set $\Omega_{0}$.

Proof. As the limit set $\Omega_{0}$ of $S_{0}$ is contained in $F_{1}$ we get that condition (ii') implies condition (ii).

Conversely, let $S_{0}=\left(F_{0}, A_{0}\right)$ be a reduced system of isometries and let $S_{n}=$ $\left(F_{n}, A_{n}\right)$ be the systems of isometries obtained by iteratively applying the Rips machine. From Proposition 3.14, $S_{n}$ is reduced. Let $P$ be an extremal point of $\operatorname{dom}\left(a_{0}\right)$ for a partial isometry $a_{0} \in A_{0}^{ \pm 1}$. By condition (ii), $P$ is in $F_{1}$ as well as $P \cdot a_{0}$, and 
thus there exists a restriction $a_{1} \in A_{1}^{ \pm 1}$ of $a_{0}$ such that $P$ is an extremal point of $\operatorname{dom}\left(a_{1}\right)$. By induction for each $n$ there exists a partial isometry $a_{n}$ in $A_{n}^{ \pm 1}$ such that $P$ is an extremal point in $\operatorname{dom}\left(a_{n}\right)$ and thus $P$ is in $F_{n}$. By Lemma 3.4 we conclude that $P$ is in the limit set $\Omega_{0}$.

\section{Computing the index of a system of isometries}

4.1. Systems of isometries on finite trees. We translate and adapt in this section Proposition 6.1 of [GLP94].

A finite tree is an $\mathbb{R}$-tree which is the convex hull of finitely many of its points. It has finitely many branch points and is the metric realization of a simplicial finite tree. A finite forest is a metric space with finitely many connected components each of which is a finite tree.

We remark that removing the branch points, such a finite forest gives a disjoint union of finitely many intervals. The integral of a function defined on $F$ is the integral on this disjoint union of intervals (with respect to Lebesgue measure).

Let $S=(F, A)$ be a system of isometries where $F$ is a finite forest. The valence of a point $P$ in $F$ is

$$
v_{S}(P)=\#\left\{a \in A^{ \pm 1} \mid P \in \operatorname{dom}(a)\right\} .
$$

We emphasize that in Section 2.3 we defined the index $i_{S}(P)$ by restricting partial isometries to the limit set $\Omega$, and thus for a system of isometries we may have $i_{S}(P)<v_{S}(P)-2$.

The function $P \mapsto v_{S}(P)$ is a finite sum of characteristic functions of finite subtrees. It is Lebesgue integrable.

We translate Proposition 6.1 of [GLP94] to get:

Proposition 4.1. Let $S=(F, A)$ be a system of isometries with independent generators. Assume that $F$ is a finite forest. Then $\int_{P \in F}\left(v_{S}(P)-2\right) \leq 0$

Proof. The sum $\int_{P \in F} v_{S}(P)$ is twice the sum of the Lebesgue measure of the domains of the partial isometries in $A$. It is denoted by $2 \ell$ in Section 6 of [GLP94] while $\int_{P \in F} 2$ is twice the Lebesgue measure of $F$ which is denoted there by $2 \mathrm{~m}$.

4.2. Shortening systems of isometries. To use Proposition 4.1 in the broader context of compact forests, we need a standard procedure to shorten a system of isometries to a finite one.

Let $F$ be a compact forest and let $\varepsilon>0$. We define:

$$
\begin{array}{r}
F_{\uparrow \varepsilon}=\left\{P \in F \mid \text { there exist } P_{-\varepsilon}, P_{+\varepsilon} \in F, P \in\left[P_{-\varepsilon}, P_{+\varepsilon}\right]\right. \\
\text { such that } \left.d\left(P, P_{-\varepsilon}\right)=d\left(P, P_{+\varepsilon}\right)=\varepsilon\right\}
\end{array}
$$


(i.e. $P$ is the midpoint of the segment $\left[P_{-\varepsilon}, P_{+\varepsilon}\right]$ which has length $2 \varepsilon$ ).

The set of extremal points $\partial F$ of a compact forest $F$ is the union of the sets of extremal points of the connected components of $F$.

Lemma 4.2. (i) For any $\varepsilon>0$, for any connected component $K$ of $F, K \cap F_{\dagger \varepsilon}=K_{\dagger \varepsilon}$ is a finite tree (possibly empty).

(ii) For any $\varepsilon>\varepsilon^{\prime}>0, F_{\uparrow \varepsilon} \subset F_{\uparrow \varepsilon^{\prime}}$.

(iii) $\bigcup_{\varepsilon>0} F_{\dagger \varepsilon}=F \backslash \partial F$.

For any partial isometry $a$ of $F$, we denote by $a_{\varepsilon}$ its restriction to $F_{\uparrow \varepsilon}$. We denote by $A_{\varepsilon}$ and $S_{\varepsilon}$ the corresponding finite set of partial isometries and the restricted system of isometries. We remark that if $S$ has independent generators then $S_{\varepsilon}$ also has.

4.3. Pseudo-surface systems of isometries. Let $S=(F, A)$ be a system of isometries, where $F$ is a compact forest and such that each point of $F$ lies inside the domain of at least two different partial isometries in $A^{ \pm 1}$. In this case the limit set $\Omega$ is equal to $F$ and the Rips machine does not do anything to $S$.

If, in addition, the system of isometries $S$ has independent generators, we say that it is pseudo-surface.

A key step in our proof of Theorem 2.2 is the following proposition which is proved using Proposition 4.1 by shortening the system of isometry as in Section 4.2. We note that the following proposition is obvious if $F$ is a finite tree or a finite forest (cf. for example [Gab96], Processus II.3 5).

Proposition 4.3. Let $S=(F, A)$ be a pseudo-surface system of isometries. Then, for any choice of three distinct partial isometries in $A^{ \pm 1}$ the intersection of the three domains contains at most one point of $F$.

Proof. By definition of pseudo-surface systems of isometries, for any $P$ in $F$ the valence $v_{S}(P)$ is greater or equal to 2 .

By contradiction we assume that there exist three distinct partial isometries $a, b$ and $c$ in $A^{ \pm 1}$ such that the intersection of their domains is strictly bigger than a point. As domains are compact subtrees, there exists a non-trivial arc $I$ which is contained in the three domains:

$$
v_{S}(P) \geq 3 \text { for all } P \in I .
$$

For any $\varepsilon>0$ we consider the finite forest $F_{\dagger \varepsilon}$ and the corresponding system of isometries $S_{\varepsilon}$.

Let $\ell$ be the length of $I$. For any $\varepsilon<\ell / 3$, the sub-arc $J$ of $I$ which is contained in the domains of the partial isometries $a_{\varepsilon}, b_{\varepsilon}$ and $c_{\varepsilon}$ of $A_{\varepsilon}$ contains the middle third of $I$ thus the length of $J$ is bigger than $\ell / 3$.

For such an $\varepsilon>0, F_{\dagger \varepsilon}$ is a finite forest by Lemma 4.2 and Proposition 4.1 holds:

$$
I_{\varepsilon}=\int_{P \in F_{\dagger \varepsilon}}\left(v_{S_{\varepsilon}}(P)-2\right) \leq 0
$$


Let $\mathcal{P}_{\varepsilon}$, resp. $\mathcal{N}_{\varepsilon}$, be the set of points of $F_{\dagger \varepsilon}$ which contributes positively, resp. negatively, to $I_{\varepsilon}$ :

$$
\mathcal{P}_{\varepsilon}=\left\{P \in F_{\uparrow \varepsilon} \mid v_{S_{\varepsilon}}(P) \geq 2\right\} \quad \text { and } \quad \mathcal{N}_{\varepsilon}=\left\{P \in F_{\uparrow \varepsilon} \mid v_{S_{\varepsilon}}(P) \leq 1\right\} .
$$

As the points in $J$ have valence at least 3, we get

$$
\int_{P \in \mathcal{P}_{\varepsilon}}\left(v_{S_{\varepsilon}}(P)-2\right) \geq \ell / 3,
$$

and thus we have

$$
0 \geq I_{\varepsilon} \geq \ell / 3+\int_{P \in \mathcal{N}_{\varepsilon}}\left(v_{S_{\varepsilon}}(P)-2\right) .
$$

Our goal is to prove that the negative part goes to zero to get a contradiction. We only need to prove that the Lebesgue measure of $\mathcal{N}_{\varepsilon}$ goes to zero.

We claim that $\mathcal{N}_{\varepsilon}$ has Lebesgue measure bounded above by $8 N^{2} \varepsilon$ where $N=\# A$ is the cardinality of $A$.

Let $P$ be in $\mathcal{N}_{\varepsilon}$, then $P$ is in $F_{\dagger \varepsilon}$ which means that $P$ is the midpoint of a segment $\left[P_{-\varepsilon}, P_{+\varepsilon}\right]$ of length $2 \varepsilon$ in $F$. As $S$ is pseudo-surface, there are at least two elements $a_{1}, a_{2} \in A^{ \pm 1}$ which are defined at $P_{-\varepsilon}$ and at least two elements $b_{1}, b_{2} \in A^{ \pm 1}$ which are defined at $P_{+\varepsilon}$. As $P$ is in $\mathcal{N}_{\varepsilon}$ at most one of the four partial isometries $a_{1 \varepsilon}, a_{2 \varepsilon}$, $b_{1 \varepsilon}, b_{2 \varepsilon}$ is defined at $P$. By switching the indices we can assume that $a_{1 \varepsilon}$ and $b_{1 \varepsilon}$ are not defined at $P$. Taking $\varepsilon$ sufficiently small ensures that the partial isometries $a_{1 \varepsilon}$ and $b_{1 \varepsilon}$ are not empty.

The domain of $a_{1 \varepsilon}$ and the point $P_{-\varepsilon}$ lie in the same connected component of $F \backslash\{P\}$ : else $P$ would be located in a segment $\left[P_{-\varepsilon}, P^{\prime}\right]$ with $P^{\prime}$ in the domain of $a_{1 \varepsilon}$ and $P_{-\varepsilon}$ in the domain of $a_{1}$, thus $P$ would be in the domain of $a_{1 \varepsilon}$. We argue similarly for the domain of $b_{1 \varepsilon}$.

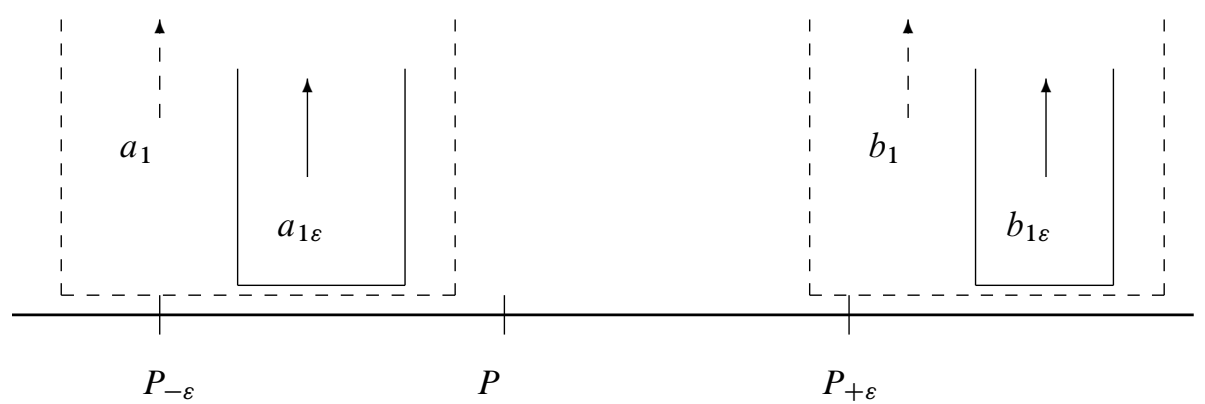

We have thus proved that $P$ is in the non-trivial arc joining the disjoint domains of $a_{1 \varepsilon}$ and $b_{1 \varepsilon}$. The point $P_{-\varepsilon}$ is in the domain of $a_{1}$ and thus at distance less than $\varepsilon$ of the domain of $a_{1 \varepsilon}$. Thus $P$ is at distance less than $2 \varepsilon$ from this domain. Hence, the length of the arc joining the disjoint domains of $a_{1 \varepsilon}$ and $b_{1 \varepsilon}$ is at most $4 \varepsilon$. 
If, over all the possible pairs of partial isometries, we sum the lengths of the arcs, we get that the volume of $\mathcal{N}_{\varepsilon}$ is bounded above by $(2 N(2 N-1) / 2) \times 4 \varepsilon$. Which proves the claim and concludes the proof.

If the compact forest $F=I$ is an interval and if the system of isometries $S=$ $(F, A)$ is pseudo-surface, Proposition 4.3 states that this is the classical case of an interval exchange transformation and $S$ is usually called surface. This justifies the terminology of pseudo-surface system of isometries.

From Proposition 4.3, it is easy to deduce a rough bound of the index of a pseudosurface system of isometries.

Corollary 4.4. Let $S=(F, A)$ be a pseudo-surface system of isometries. Then $i(S)$ is finite and bounded above by a constant depending only on the cardinality of $A$.

Proof. We denote, as before, by $N=\# A$ the cardinal of $A$. From the previous proposition, there are at most $\left(\begin{array}{c}2 N \\ 3\end{array}\right)$ points in $F$ which belongs to the domains of at least three different partial isometries in $A^{ \pm 1}$. Each of these points has valence at most $2 N$. Adding up we get that

$$
i(S)=\sum_{P \in F} i_{S}(P)=\sum_{P \in F}\left(v_{S}(P)-2\right) \leq N(2 N-1)(2 N-2)^{2} / 3 .
$$

We now state a combinatorial lemma.

Let $K$ be a compact $\mathbb{R}$-tree and let $\mathcal{K}=\left(K_{a}\right)_{a \in A}$ be a finite collection of compact subtrees of $K$. For such a collection, and for any point $P \in K$ we denote by $v_{\mathcal{K}}(P)$ the number of elements $a$ of $A$ such that $P$ is in $K_{a}$.

Lemma 4.5. Let $\mathcal{K}=\left(K_{a}\right)_{a \in A}$ be a finite collection of compact subtrees of a compact $\mathbb{R}$-tree $K$. Assume that

(1) for any choice of three distinct elements of A the intersection of the corresponding subtrees is at most one point,

(2) any element $P$ of $K$ is in at least two compact subtrees $K_{a}, K_{b}(a \neq b \in A)$. Then

$$
\sum_{P \in K}\left(v_{\mathcal{K}}(P)-2\right)=\# A-2
$$

Proof. Let $T$ be the convex hull in $K$ of all elements $P \in K$ such that $v_{\mathcal{K}}(P) \geq 3$. From our first hypothesis $T$ is a finite tree. For each $a \in A$ the intersection $T_{a}=$ $K_{a} \cap T$ is a finite tree. Let $\mathcal{T}=\left(T_{a}\right)_{a \in A}$ be the corresponding collection of finite subtrees of $T$. We have the equality

$$
\sum_{P \in K}\left(v_{\mathcal{K}}(P)-2\right)=\sum_{P \in T}\left(v_{\mathcal{T}}(P)-2\right) .
$$


Moreover $\mathcal{T}$ satisfies the same hypothesis as $\mathcal{K}$ : the intersection of three of its elements is at most a point and any element of $T$ is in at least two subtrees $T_{a}$ and $T_{b}, a \neq b \in A$. We regard $T$ and each $T_{a}$ as a simplicial tree by considering all the branch points and extremal points as vertices. Each edge of the simplicial tree $T$ belongs to exactly two trees $T_{a}$ and $T_{b}$. Combinatorial computation gives

$$
\begin{aligned}
\sum_{P \in T}\left(v_{\mathcal{T}}(P)-2\right) & =\sum_{P \in V(T)}\left(v_{\mathcal{T}}(P)-2\right)=\sum_{P \in V(T)} \sum_{a \in A} \mathbb{1}_{P \in V\left(T_{a}\right)}-2 \# V(T) \\
& =\sum_{a \in A} \sum_{P \in V(T)} \mathbb{1}_{P \in V\left(T_{a}\right)}-2 \# E(T)-2 \\
& =\sum_{a \in A} \# V\left(T_{a}\right)-\sum_{a \in A} \# E\left(T_{a}\right)-2 \\
& =\# A-2 .
\end{aligned}
$$

We can now get the correct bound for the index of a pseudo-surface system of isometries

Theorem 4.6. Let $S=(F, A)$ be a pseudo-surface system of isometries and, let $\Gamma$ be the associated graph. Then $i(S)=i(\Gamma)$.

Proof. As $S$ is pseudo-surface, at least two distinct partial isometries are defined at each point of $F$. Thus, the graph $\Gamma$ does not have vertices of valence 0 or 1 and its index is given by

$$
i(\Gamma)=\sum_{K \in V(\Gamma)} i_{\Gamma}(K) .
$$

Let $K$ be a connected component of $F$ and let $B$ be the subset of $A^{ \pm 1}$ which consists of partial isometries with domains inside $K$. The set $B$ is also the set of edges going out of the vertex $K$ of the graph $\Gamma$ and thus

$$
i_{\Gamma}(K)=\# B-2 \text {. }
$$

Let $\mathcal{K}$ be the collection of domains of elements of $B$. Thus for each point $P$ in $K$

$$
i_{S}(P)=v_{\mathcal{K}}(P)-2 .
$$

By Proposition 4.3, the collection $\mathcal{K}$ satisfies the hypothesis of Lemma 4.5 and we get

$$
\sum_{P \in K}\left(v_{\mathcal{K}}(P)-2\right)=\# B-2 .
$$

Thus the contribution of the points of $K$ to the index of $S$ is equal to the contribution of the corresponding vertex of $\Gamma$ :

$$
\sum_{P \in K} i_{S}(P)=i_{\Gamma}(K)
$$

Adding up for all connected components $K$ of $F$, proves the theorem. 
4.4. Proof of Theorem 2.2. Using Proposition 3.6, Theorem 2.2 is a consequence of the following result.

Proposition 4.7. Let $S=(F, A)$ be a system of isometries with independent generators. Let $\hat{\Gamma}$ be its limit graph. Then the index of $S$ is equal to the index of $\hat{\Gamma}$ :

$$
i(S)=i(\widehat{\Gamma}) .
$$

Proof. Let $\Omega_{0}$ be the union of all non-trivial connected components of the limit set $\Omega$. By Proposition 3.9, $\Omega_{0}$ has finitely many connected components, that is to say, $\Omega_{0}$ is a compact forest. Let $S_{0}=\left(\Omega_{0}, A_{0}\right)$ be the system of isometries which consists of the restrictions of $S$ to $\Omega_{0}$. By definition of the limit set, $S_{0}$ is a pseudo-surface system of isometries. By Proposition 4.3, the intersection the domains of three distinct partial isometries of $A_{0}^{ \pm 1}$ contains at most one point.

For a vertex of $\hat{\Gamma}$ corresponding to a connected component $K$ (possibly a single point) of $\Omega$ we can apply Lemma 4.5 to the collection given by the domains of the edges going out of $K$ to get:

$$
i_{\widehat{\Gamma}}(K)=\sum_{P \in K}\left(v_{S}(P)-2\right) .
$$

By Corollary 3.7, $\widehat{\Gamma}$ has finitely many vertices with index strictly positive (and these indices are finite). Adding up for all these singular vertices of $\widehat{\Gamma}$, we get

$$
i(S)=i(\widehat{\Gamma}) \text {. }
$$

4.5. Systems of isometries with maximal index. From Theorem 2.2 , we say that a system of isometries $S$ has maximal index if its index is equal to the index of its associated graph $\Gamma: i(S)=i(\Gamma)$.

The following proposition characterizes reduced systems of isometries with maximal index.

Theorem 4.8. Let $S=(F, A)$ be a reduced system of isometries with independent generators, let $\Gamma$ be its associated graph, and $\widehat{\Gamma}$ be its limit graph. The following are equivalent:

(1) The system of isometries $S$ has maximal index.

(2) The graph $\widehat{\Gamma}$ is finite.

(3) The Rips machine, starting from $S$, halts after finitely many steps.

Proof. As before we denote by $S_{n}=\left(F_{n}, A_{n}\right)$ the system of isometries obtained after $n$ steps of the Rips machine. By Proposition 3.13 and Proposition 3.14 the Rips machine only performs "split an edge" moves and induces a homotopy equivalence $\tau_{n}: \Gamma_{n+1} \rightarrow \Gamma_{n}$ at each step. And thus the index is constant:

$$
i\left(\Gamma_{n}\right)=i\left(\Gamma_{0}\right)=i(\Gamma) .
$$


Moreover at each step the graph $\Gamma_{n}$ does not have vertices of valence 0 or 1 .

$3 \Longrightarrow 2$ : If the Rips machine halts after step $n$, then $\Gamma_{n+1}=\Gamma_{n}=\widehat{\Gamma}$ is a finite graph.

$2 \Longrightarrow 3$ : Conversely, at each step $n$ the Rips machine only performs "split an edge" moves. This move adds one edge to $\Gamma_{n}$ to get $\Gamma_{n+1}$. If the Rips machine never halts, then the number of edges of $\Gamma_{n}$ goes to infinity. As each of the $\tau_{n}$ is onto, we get that $\widehat{\Gamma}$ is infinite.

$3 \Longrightarrow 1$ : If the Rips machine halts after finitely many steps: for $n$ big enough

$$
\widehat{\Gamma}=\Gamma_{n+1}=\Gamma_{n},
$$

by Proposition 4.7, $i(\widehat{\Gamma})=i(S)$ and we get that $S$ has maximal index.

$1 \Longrightarrow 3$ : Assume that $S$ has maximal index: $i(S)=i(\widehat{\Gamma})=i(\Gamma)$.

We proceed as in the proof of Proposition 3.6. Let $\Theta_{0}$ be the finite subset of vertices of $\widehat{\Gamma}$ with valence strictly bigger than 2 . Let $\Theta$ be the finite subgraph of $\widehat{\Gamma}$ which contains all edges incident to $\Theta_{0}$. The graph $\Theta$ contains all the index of $\hat{\Gamma}$ :

$$
i(\widehat{\Gamma})=\sum_{x \in \Theta_{0}} i_{\Theta}(x) .
$$

For $n$ big enough, $\hat{\tau}_{n}$ is injective on $\Theta$ and thus for each vertex $x$ in $\Theta_{0}$

$$
i_{\Theta}(x) \leq i_{\Gamma_{n}}\left(\hat{\tau}_{n}(x)\right) .
$$

We assumed that $S$ is reduced and thus that $\Gamma_{n}$ does not have vertices of strictly negative index.

By maximality of the index, $i(\widehat{\Gamma})=i\left(\Gamma_{n}\right)$ and thus we can compute

$$
i(\hat{\Gamma})=\sum_{x \in \Theta_{0}} i_{\Theta}(x)=\sum_{x \in \Theta_{0}} i_{\Gamma_{n}}\left(\hat{\tau}_{n}(x)\right)+\sum_{y \in V\left(\Gamma_{n}\right) \backslash \hat{\tau}_{n}\left(\Theta_{0}\right)} i_{\Gamma_{n}}(y)=i\left(\Gamma_{n}\right) .
$$

We deduce that for each $x$ in $\Theta_{0}$ and for all $y \in V\left(\Gamma_{n}\right) \backslash \hat{\tau}_{n}\left(\Theta_{0}\right)$,

$$
i_{\Theta}(x)=i_{\Gamma_{n}}\left(\hat{\tau}_{n}(x)\right) \text { and } i_{\Gamma_{n}}(y)=0 .
$$

The commutative diagram

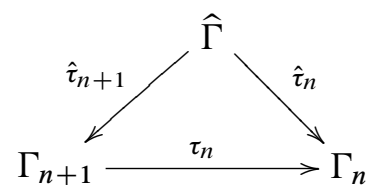

restricts to graph isomorphisms between $\Theta$ and its images. Moreover, $\hat{\tau}_{n+1}\left(\Theta_{0}\right)$ and $\hat{\tau}_{n}\left(\Theta_{0}\right)$ contain all the vertices of strictly positive index of $\Gamma_{n+1}$ and $\Gamma_{n}$ respectively. Thus no "split an edge" move can occur when passing from $\Gamma_{n}$ to $\Gamma_{n+1}$ and thus the Rips machine does not do anything to the system of isometries $S_{n}$. 


\section{Trees}

Throughout this section, $T$ is an $\mathbb{R}$-tree with a very small, minimal action of the free group $\mathbb{F}_{N}$ of rank $N$ by isometries with dense orbits.

5.1. The map $\mathcal{Q}$. Let $P$ be a point in $T$, we consider the equivariant map $\mathcal{Q}_{P}: \mathbb{F}_{N} \rightarrow$ $T, u \mapsto u \cdot P$. This maps does not extends continuously to the boundary $\partial \mathbb{F}_{N}$ of $\mathbb{F}_{N}$. To overcome this difficulty we weaken the topology on $T$ by considering the observers' topology.

Let $\widehat{T}=\bar{T} \cup \partial T$ be the union of the metric completion of $T$ and its (Gromov) boundary. $\widehat{T}$ inherits from the metric on $T$ a well defined topology. However, $\hat{T}$ is not compact in general.

We consider on $\widehat{T}$ the weaker observers' topology and we denote by $\widehat{T}^{\text {obs }}$ this topological space. A basis of open sets in $\widehat{T}^{\text {obs }}$ is given by the set of connected components of $\widehat{T} \backslash\{P\}$ for all points $P$. This topology is Hausdorff and $\widehat{T}^{\text {obs }}$ is a compact space with the same connected subspaces than $\widehat{T}$, see [CHL07].

Proposition 5.1 ([CHL07]). Let $T$ be an $\mathbb{R}$-tree with a very small, minimal action of $\mathbb{F}_{N}$ by isometries with dense orbits. There exists a unique map $\mathbb{Q}$ that is the continuous extension from $\partial \mathbb{F}_{N}$ to $\widehat{T}^{\text {obs }}$ of the map $\mathcal{Q}_{P}: u \mapsto u \cdot P$. The map $\mathcal{Q}$ does not depend on the choice of a point $P$.

This map $\mathcal{Q}$ was first introduced by Levitt and Lustig in [LL03], [LL08] with a slightly different approach. In particular they proved

Proposition 5.2. Let $T$ be an $\mathbb{R}$-tree with a very small, minimal action of $\mathbb{F}_{N}$ by isometries with dense orbits. The map $\mathcal{Q}$ is onto $\widehat{T}$. The points $P$ in $\widehat{T}$ with strictly more than one pre-image by $\mathcal{Q}$ are in the metric completion $\bar{T}$ of $T$ (and not in the (Gromov) boundary $\partial T$ ).

It has been asked by Levitt and Lustig ([LL03], Remark 3.6) whether the map $\mathcal{Q}: \partial \mathbb{F}_{N} \rightarrow \widehat{T}$ has finite fibers (in the case where the action is free). We are going to answer this question and to give a precise bound for the cardinal of the fibers. In this purpose we need to make this question precise by the following definition of the Q-index.

5.2. The $\mathcal{Q}$-index. We denote by $\operatorname{Stab}(P)$ the stabilizer in $\mathbb{F}_{N}$ of a point $P$ of $\widehat{T}$.

It is proved in [GL95] that $\operatorname{Stab}(P)$ is a finitely generated subgroup of $\mathbb{F}_{N}$. The subgroup $\operatorname{Stab}(P)$ is a free group and its boundary $\partial \operatorname{Stab}(P)$ embeds in the boundary of $\mathbb{F}_{N}$. For any element $X \in \partial \operatorname{Stab}(P) \subseteq \partial \mathbb{F}_{N}$, Proposition 5.1 proves that $Q(X)=$ $P$. Elements of $\partial \operatorname{Stab}(P)$ are called singular, and the other elements of the fiber $Q^{-1}(P)$ are regular. We denote by $\mathcal{Q}_{r}^{-1}(P)$ the set of regular points. As $\mathcal{Q}$ is equivariant, $\operatorname{Stab}(P)$ acts on $Q^{-1}(P)$ and on $Q_{r}^{-1}(P)$. 
The $Q$-index $\operatorname{ind}_{\mathcal{Q}}(P)$ of a point $P$ in $\widehat{T}$ is defined by

$$
\operatorname{ind}_{\mathcal{Q}}(P)=\#\left(\mathcal{Q}_{r}^{-1}(P) / \operatorname{Stab}(P)\right)+2 \operatorname{rank}(\operatorname{Stab}(P))-2 .
$$

When $\operatorname{Stab}(P)$ is trivial this definition becomes

$$
\operatorname{ind}_{\mathcal{Q}}(P)=\# Q^{-1}(P)-2 .
$$

The $\mathcal{Q}$-index only depends on the orbit $[P]$ of $P$ under the action of $\mathbb{F}_{N}$ and we can define the $\mathcal{Q}$-index of the tree $T$ by

$$
\operatorname{ind}_{\mathcal{Q}}(T)=\sum_{[P] \in \widehat{T} / \mathbb{F}_{N}} \max \left(0 ; i_{\mathcal{Q}}([P])\right) .
$$

From Proposition 5.2, points in $\partial T$ have exactly one pre-image by $\mathcal{Q}$. Thus, only points in $\bar{T}$ contribute to the $\mathcal{Q}$-index of $T$.

The main goal of this section is to prove the following theorem:

Theorem 5.3. Let $T$ be an $\mathbb{R}$-tree with a very small, minimal action of $\mathbb{F}_{N}$ by isometries with dense orbits. Then

$$
\operatorname{ind}_{\mathcal{Q}}(T) \leq 2 N-2
$$

In the case of a free action of the free group $\mathbb{F}_{N}$ on $T$ this gives the answer to Levitt and Lustig's question:

Corollary 5.4. Let $T$ be an $\mathbb{R}$-tree with a free, minimal action of $\mathbb{F}_{N}$ by isometries with dense orbits. Then, there are finitely many orbits of points $P$ in $\hat{T}$ with strictly more than 2 elements in their $\mathcal{Q}$-fiber $\mathcal{Q}^{-1}(P)$ and these fibers are finite.

5.3. Dual lamination and compact heart. The double boundary of $\mathbb{F}_{N}$ is

$$
\partial^{2} \mathbb{F}_{N}=\left(\partial \mathbb{F}_{N} \times \partial \mathbb{F}_{N}\right) \backslash \Delta
$$

where $\Delta$ is the diagonal. An element of $\partial^{2} \mathbb{F}_{N}$ is a line.

Using the map $\mathcal{Q}$, in [CHL08], the dual lamination $L(T)$ to the tree $T$ is defined:

$$
L(T)=\left\{(X, Y) \in \partial^{2} \mathbb{F}_{N} \mid \mathcal{Q}(X)=\mathcal{Q}(Y)\right\} .
$$

From this definition, the map $\mathcal{Q}$ naturally induces an equivariant map $\mathcal{Q}^{2}: L(T) \rightarrow$ $\widehat{T}$. It is proved in [CHL08] that the map $\mathcal{Q}^{2}$ is continuous and its image is a subset $\Omega$ of $\bar{T}$ which we call the limit set.

We fix a basis $A$ of $\mathbb{F}_{N}$. Elements of $\mathbb{F}_{N}$ are reduced finite words in the alphabet $A^{ \pm 1}$. An element $X$ of $\partial \mathbb{F}_{N}$ is an infinite reduced words in $A^{ \pm 1}$, we denote by $X_{1}$ its first letter. 
The unit cylinder $C_{A}(1)$ of $\partial^{2} \mathbb{F}_{N}$ is

$$
C_{A}(1)=\left\{(X, Y) \in \partial^{2} \mathbb{F}_{N} \mid X_{1} \neq Y_{1}\right\}
$$

Although $\partial^{2} \mathbb{F}_{N}$ is not compact, the unit cylinder is compact and indeed a Cantor set.

In [CHL09] the relative limit set of $T$ with respect to $A$ is defined:

$$
\Omega_{A}=Q^{2}\left(L(T) \cap C_{A}(1)\right) .
$$

From the continuity of the map $\mathcal{Q}^{2}$, the relative limit set $\Omega_{A}$ is a compact subset of $\bar{T}$.

The compact heart $K_{A}$ of $T$ is the convex hull of $\Omega_{A}$.

For any element $a$ of the basis $A$ we consider the partial isometry (which we also denote by $a$, but which we write on the right) which is the restriction of the action of $a^{-1}$ :

$$
K_{A} \cap a K_{A} \rightarrow K_{A} \cap a^{-1} K_{A}, \quad x \mapsto x \cdot a=a^{-1} x .
$$

We get a system of isometries $S_{A}=\left(K_{A}, A\right)$ as defined in Section 2 .

In [CHL09] it is proved that $S$ encodes all the informations given by $T$ and the action of $\mathbb{F}_{N}$. To be more specific, we summarize results of [CHL09] as follows:

Proposition 5.5 ([CHL09]). Let $T$ be an $\mathbb{R}$-tree with a very small, minimal action of $\mathbb{F}_{N}$ by isometries with dense orbits. Let $A$ be a basis of $\mathbb{F}_{N}$, let $K_{A}$ be its compact heart and $S_{A}=\left(K_{A}, A\right)$ be the associated system of isometries. Then

(1) $S_{A}$ has independent generators.

(2) For any word $u \in \mathbb{F}_{N}$, and for any point $P \in K_{A}, u^{-1} P \in K_{A}$ if and only if $u$ is admissible for $S_{A}$ and $P \in \operatorname{dom}(u)$. In this case $P \cdot u=u^{-1} \cdot P$.

(3) For any element $X \in \partial \mathbb{F}_{N}, \mathcal{Q}(X)=P \in K_{A}$ if and only if $X$ is admissible and $\{P\}=\operatorname{dom}(X)$.

Proof. Assertion 1 is Lemma 5.1 of [CHL09]. Assertion 2 is proved in Lemma 3.5 (1) and Corollary 5.5 and Assertion 3 is a consequence of Proposition 4.3, Lemma 4.7 and Corollary 5.5.

We deduce that for an infinite reduced admissible word $X$ the definition of $\mathcal{Q}(X)$ of Section 2.1 agrees with the definition given by Proposition 5.1. Moreover the relative limit set $\Omega_{A}$ of the $\mathbb{R}$-tree $T$ is equal to the limit set of the system of isometries $S_{A}$.

5.4. The compact heart of a tree is reduced. As explained in Section 3.4, reduced systems of isometries (see Definition 3.11) considerably simplifies the use of the Rips machine. Fortunately, in the context of $\mathbb{R}$-trees, which we are studying, and thanks to [CHL09], we can work with reduced systems of isometries.

Proposition 5.6. Let $T$ be an $\mathbb{R}$-tree with a minimal very small action of $\mathbb{F}_{N}$ by isometries with dense orbits. Let $A$ be a basis of $\mathbb{F}_{N}$, let $\Omega_{A}$ be the relative limit 
set and $K_{A}$ be the compact heart of $T$. Let $S_{A}=\left(K_{A}, A\right)$ the induced system of isometries.

Then the system of isometries $S_{A}$ is reduced.

Proof. By Proposition 5.2, the map $\mathcal{Q}$ is onto $\widehat{T}$ : for any point $P$ in $K_{A}$ there exists $X \in \partial \mathbb{F}_{N}$ such that $Q(X)=P$. By Proposition 5.5, $X$ is admissible and $P$ is in the domain of $X$. Thus, any point $P$ of the compact heart $K_{A}$ has an infinite tree of trajectories.

Let $S_{1}=\left(F_{1}, A_{1}\right)$ be the output of the Rips machine. Let $a$ be a partial isometry in $A$ and let $P$ be an extremal point of the domain of $a$. If $P$ is an extremal point of $K_{A}$, then, as $K_{A}$ is the convex hull of the relative limit set $\Omega_{A}$, we get that $P$ is in $\Omega_{A}$ and thus in $F_{1}$. If $P$ is not an extremal point of $K_{A}$ there exists a sequence $Q_{n}$ of points in $F$ which converges to $P$ and which are not in the domain of $a$. The points in the compact heart $K_{A}$ have infinite tree of trajectories, thus for each of these points $Q_{n}$ there exists a partial isometry $b_{n}$ in $A$ such that $Q_{n}$ is in the domain of $b_{n}$. As $A$ is finite, up to passing to a subsequence we assume that all the $Q_{n}$ are in the domain of a partial isometry $b$ in $A$. The domain of $b$ is close and thus $P$ is in the domain of $b$. By definition of the Rips machine we get that $P$ is in $F_{1}$.

5.5. Proof of Theorem 5.3. Theorem 5.3 is a consequence of Theorem 2.2 and of the following theorem which relates the $\mathcal{Q}$-index of $T$ and the index of the system of isometries $S_{A}$ defined on its compact heart.

Theorem 5.7. Let $T$ be an $\mathbb{R}$-tree with a very small, minimal action of $\mathbb{F}_{N}$ by isometries with dense orbits. Let $A$ be a basis of $\mathbb{F}_{N}$. The Q $Q$-index of $T$ and the index of the induced system of isometries $S_{A}=\left(K_{A}, A\right)$ on the heart $K_{A}$ of $T$ and $A$ are equal:

$$
\operatorname{ind}_{\mathcal{Q}}(T)=i\left(S_{A}\right) .
$$

Proof. Let $P$ be a point in $\widehat{T}$ and $[P]$ be its orbit under the action of $\mathbb{F}_{N}$. By Proposition 5.5, assertion 2, the intersection of the orbit $[P]$ and of the compact heart $K_{A}$ is a pseudo-orbit (possibly empty) of the system of isometries $S_{A}$.

Assume that $\operatorname{ind}_{\mathcal{Q}}([P]) \geq 0$. There are at least two distinct elements $X, Y$ in the fiber $Q^{-1}(P)$. Let $u$ be the common prefix of $X$ and $Y$, then $X^{\prime}=u^{-1} X$ and $Y^{\prime}=u^{-1} Y$ have different first letter and are in the pre-image by $\mathcal{Q}$ of $P^{\prime}=u^{-1} P$. By definition $P^{\prime}$ is in the relative limit set $\Omega_{A}$ of the tree $T$.

This proves that the $\mathcal{Q}$-index of $T$ can be computed by considering only pseudoorbits in $K_{A}$ :

$$
\operatorname{ind}_{\mathcal{Q}}(T)=\sum_{[P] \in K_{A} / \mathbb{F}_{N}} \max \left(0 ; \operatorname{ind}_{\mathcal{Q}}([P])\right) .
$$

Let $P$ be a point in $K_{A}$. By Proposition 5.5, the boundary at infinity of the tree 
of trajectories $\widetilde{\Gamma}(P)$ is exactly $\mathcal{Q}^{-1}(P)$ and the discussion in Section 2.4 shows that

$$
\begin{aligned}
\operatorname{ind}_{\mathcal{Q}}([P]) & =\#\left(\mathcal{Q}_{r}^{-1}(P) / \operatorname{Stab}(P)\right)+2 \operatorname{rank}(\operatorname{Stab}(P))-2 \\
& =\# \partial \Gamma(P)+2 \operatorname{rank}(\operatorname{Stab}(P))-2 \\
& =\sum_{P^{\prime} \in[P] \cap K_{A}} i_{S}\left(P^{\prime}\right) .
\end{aligned}
$$

Adding up for all points $P$ in $K_{A}$, proves the theorem.

5.6. Geometric index of a tree. Gaboriau and Levitt in [GL95] introduced the index of $T$ as follows.

Let $P$ a point in $T$ and let $\pi_{0}(T \backslash\{P\})$ be the set of connected components of $T$ without $P$. The stabilizer of $P$ acts on this set. The geometric index of $P$ is

$$
\operatorname{ind}_{\mathrm{geo}}(P)=\#\left(\pi_{0}(T \backslash\{P\}) / \operatorname{Stab}(P)\right)+2 \operatorname{rank}(\operatorname{Stab}(P))-2 .
$$

This index is always non-negative because there are no terminal vertices in a minimal tree. If the action of $\mathbb{F}_{N}$ on the tree $T$ is free the above definition becomes simpler:

$$
\operatorname{ind}_{\text {geo }}(P)=\# \pi_{0}(T \backslash\{P\})-2 .
$$

The geometric index is constant inside an orbit under the action of $\mathbb{F}_{N}$. The geometric index of $T$ is then the sum of the indices over all orbits of points:

$$
\operatorname{ind}_{\text {geo }}(T)=\sum_{[P] \in T / \mathbb{F}_{N}} \operatorname{ind}_{\text {geo }}(P)
$$

The following theorem is proved by Gaboriau and Levitt:

Theorem 5.8 ([GL95]). The geometric index of an $\mathbb{R}$-tree with a very small minimal action of the free group $\mathbb{F}_{N}$ is bounded above by $2 N-2$.

5.7. Botanic of trees. In this section we establish a beginning of classification of trees in the boundary of Outer Space. Let $T$ be an $\mathbb{R}$-tree with a minimal very small action of $\mathbb{F}_{N}$ by isometries with dense orbits. Let $A$ be a basis of $\mathbb{F}_{N}$, let $\Omega_{A}$ be the relative limit set, let $K_{A}$ be the compact heart of $T$ and let $S_{A}=\left(K_{A}, A\right)$ be the associated system of isometries.

We first recall the existing terminology of geometric trees. The tree $T$ is geometric if it can be obtained from a system of isometries on a finite tree, as explained in the Introduction (see for instance [Gab97, Bes02]). Geometric trees can be alternatively characterized thanks to the following:

Theorem 5.9 ([GL95], see also Corollary 6.1 in [CHL09]). Let $T$ be an $\mathbb{R}$-tree with a minimal, very small action by isometries of $\mathbb{F}_{N}$ with dense orbits. The following are equivalent: 
(1) $T$ is geometric;

(2) the geometric index is maximal: $\operatorname{ind}_{\mathrm{geo}}(T)=2 N-2$;

(3) $K_{A}$ is a finite tree.

We now introduce more terminology. The tree $T$ is of surface type if the Rips machine, starting with the system of isometries $S_{A}=\left(K_{A}, A\right)$, halts after finitely many steps. More precisely, a tree of surface type is:

- a surface tree if it is geometric (this terminology is justified by the fact that a tree dual to measured foliation on a surface with boundary is a surface tree.),

- a pseudo-surface tree if it is not geometric.

(Note that the fact that a tree is pseudo-surface does not exactly correspond to the fact that the system of isometries $S_{A}=\left(K_{A}, A\right)$ is pseudo-surface, according to the definition given in Section 4.3).

By Theorem 4.8 and Theorem 5.7 we get the following characterization of trees of surface type:

Theorem 5.10. Let $T$ be an $\mathbb{R}$-tree with a minimal, very small action by isometries of $\mathbb{F}_{N}$ with dense orbits. The tree $T$ is of surface type if and only if its $\mathcal{Q}$-index is maximal: $\operatorname{ind}_{\mathcal{Q}}(T)=2 N-2$.

This proves in particular that being of surface type is a property of $T$ and does not depend on the choice of a basis $A$ of the free group $\mathbb{F}_{N}$.

The tree $T$ is of Levitt type if its relative limit set $\Omega_{A}$ is totally disconnected (i.e. the connected components of $\Omega_{A}$ are points). More precisely, a tree of Levitt type is

- Levitt if it is geometric (these trees were discovered by Levitt [Lev93] and are also termed thin or exotic).

- pseudo-Levitt if it is not geometric.

We now prove that being of Levitt type is a property of $T$ and does not depend on the choice of a basis $A$ of $\mathbb{F}_{N}$.

Let $L(T)$ be the dual lamination of the tree $T$. The limit set $\Omega$ of $T$ is the image in the metric completion $\bar{T}$ of $L(T)$ by the map $\mathcal{Q}^{2}$ :

$$
\Omega=Q^{2}(L(T)) .
$$

Contrary to the relative limit set $\Omega_{A}$, the limit set $\Omega$ is in general not closed.

Theorem 5.11. The tree $T$ is of Levitt type if and only if the limit set $\Omega$ is totally disconnected.

Proof. By definition, the relative limit set $\Omega_{A}=\mathcal{Q}^{2}\left(L(T) \cap C_{A}(1)\right)$ is a subset of $\Omega$. Thus, if $\Omega$ does not contain a non-trivial arc, $\Omega_{A}$ neither. 
Conversely, the double boundary of $\mathbb{F}_{N}$ is the union of the translates of the unit cylinder and

$$
L(T)=\bigcup_{u \in \mathbb{F}_{N}} u\left(L(T) \cap C_{A}(1)\right) \quad \text { and } \quad \Omega=\bigcup_{u \in \mathbb{F}_{N}} u \Omega_{A}
$$

In particular if $I$ is a non-trivial arc in the limit set $\Omega$, it is the countable union of its intersections with translates of the relative limit set $\Omega_{A}$. Using Baire's Property for $I$, we get that $\Omega_{A}$ contains a non-trivial arc.

We remark that there are trees in the boundary of Outer Space which are neither of surface or Levitt type. These are trees of mixed type, that is to say their relative limit set $\Omega_{A}$ contains non-trivial arcs but have infinitely many connected components.

Remark 5.12. A general classification of systems of isometries, in particular a Theorem à-la Imanishi to decompose trees of mixed type would be of interest. More generally, the question of understanding the relationships between mixing properties of trees, indecomposability of systems of isometries and minimality of laminations seems to be natural. Together with Reynolds [CHR11] we prove that indecomposable trees and minimal (up to diagonal leaves) laminations are dual to each other. In this spirit, see also the work of Reynolds [Rey10].

5.8. Mixing trees. In this section we give sufficient hypothesis on a tree to enforce that it is either of surface type or of Levitt type.

We first describe the limit set of trees of surface type.

Proposition 5.13. Let $T$ be an $\mathbb{R}$-tree with a minimal, very small action by isometries of $\mathbb{F}_{N}$ with dense orbits. If the tree $T$ is of surface type, then the limit set $\Omega$ is connected and contains $T$.

Proof. As $T$ is minimal, and as $\Omega$ is $\mathbb{F}_{N}$-invariant, we get that $\Omega$ is connected if and only if $\Omega$ contains $T$ :

$$
T \subseteq \Omega \subseteq \bar{T} .
$$

If $T$ is of surface type, the Rips machine starting with the system of isometries $S_{A}=\left(K_{A}, A\right)$, halts after finitely many steps, and thus $\Omega_{A}=F_{n}$ for some $n$, where $F_{n}$ is the forest remaining after $n$ steps of the Rips machine. The system of isometries is reduced, hence the pseudo-orbit of each point in $K_{A}$ is infinite and thus meets $F_{n}$, which proves that $K_{A} \subseteq \mathbb{F}_{N} . \Omega_{A}$. Moreover the orbit of each point in $T$ meets $K_{A}$, thus $T \subseteq \mathbb{F}_{N} K_{A}$, which concludes the proof.

A converse of this proposition that requires stronger hypothesis on $T$ is proved in Proposition 5.14.

A segment of an $\mathbb{R}$-tree is a subset isometric to a compact real interval which is not reduced to a point. The action of $\mathbb{F}_{N}$ on an $\mathbb{R}$-tree $T$ by isometries is arc-dense if 
every segment of $T$ meets every orbit. Following [Mor88], the action is mixing if for every segments $I$ and $J$ in $T$, the segment $J$ is covered by finitely many translates of $I$ : there exists finitely many elements $u_{1}, \ldots, u_{r}$ of $\mathbb{F}_{N}$ such that

$$
J \subseteq u_{1} I \cup \cdots \cup u_{r} I .
$$

Finally, Guirardel [Gui08] defined an action to be indecomposable, if for every segments $I$ and $J$ in $T$, the segment $J$ is covered by finitely many overlapping translates of $I$ : there exists finitely many elements $u_{1}, \ldots, u_{r}$ of $\mathbb{F}_{N}$ such that

$$
J \subseteq u_{1} I \cup \cdots \cup u_{r} I \text { and } u_{i} \cap u_{i+1} \text { is a segment, } i=1, \ldots, r-1 .
$$

It is obvious that an indecomposable action is mixing and, that a mixing action is arc-dense. An arc-dense action has dense orbits and is minimal.

Proposition 5.14. Let $T$ be an $\mathbb{R}$-tree with a mixing action of $\mathbb{F}_{N}$ by isometries. Then $T$ is either of surface type or of Levitt type.

Proof. Let $A$ be a basis of $\mathbb{F}_{N}$, let $\Omega_{A}$ be the relative limit set, $K_{A}$ the compact heart of $T$ and $S_{A}=\left(K_{A}, A\right)$ be the associated system of isometries.

By contradiction assume that $T$ is neither of surface type or of Levitt type. Then by definition of Levitt type, $\Omega_{A}$ contains a non-trivial connected component and thus a segment $I$.

Let $S_{0}=S_{A}$ and let $S_{n}=\left(F_{n}, A_{n}\right)$ be the sequence of systems of isometries obtained from $S_{A}$ by applying the Rips machine. By definition of surface type, the Rips machine runs forever.

Let $E_{0}$ be the set of points of $F_{0}=K_{A}$ erased at the first step of the Rips machine: $E_{0}=F_{0} \backslash F_{1}$. As $S_{0}$ is reduced, $E_{0}$ is contained in the convex hull in $F_{0}$ of $F_{1}$ and is a finite union of finite trees and an open subset of $F_{0}$.

Let $E_{n}=F_{n} \backslash F_{n+1}$ be the subset of $F_{n}$ erased at the $n+1$ step of the Rips machine. Let $D_{n}$ be the subset of $E_{0}$ defined by

$$
D_{n}=\left\{P \in E_{0} \mid \text { there is } u \in \mathbb{F}_{N},|u|=n \text {, and } P u_{i} \in E_{i} \text {, for } i=1, \ldots, n\right\}
$$

where $u_{i}$ is the prefix of $u$ of length $i$. By definition, for each $n, D_{n+1} \subset D_{n}$. As the Rips machine runs forever, $D_{n}$ is a non-empty subset of $F_{0}$. We distinguish two cases.

First assume that the nested intersection of the open non-empty subsets $D_{n}$ is non-empty and let $P_{0} \in \bigcap_{n \in \mathbb{N}} D_{n}$. As $P_{0}$ is in the open subset $E_{0}$ it is not an extremal point of $F$ and as $T$ is arc-dense, there exists $u \in \mathbb{F}_{N}$ such that $u P_{0} \in I$. From Proposition 5.5, the partial isometry $u^{-1}$ is defined at $P_{0}$ and $P_{0} u^{-1}=u P_{0}$ is in the relative limit set $\Omega_{A}$. By definition of the Rips machine, for $n$ bigger than $|u|$, $P_{0}$ is not in $D_{n}$. A contradiction. 
Assume now that the nested intersection $\bigcap_{n \in \mathbb{N}} D_{n}$ is empty and let $P_{0}$ be in the nested intersection of compact subsets

$$
P_{0} \in \bigcap_{n \in \mathbb{N}} \bar{D}_{n}
$$

Then, there exists $n_{0}$, such that for $n$ bigger than $n_{0}, P_{0}$ is in $\bar{D}_{n} \backslash D_{n}$. Recall that $\bar{D}_{n_{0}}$ is a finite tree and let $Q$ be a point of $D_{n_{0}}$. Then $Q$ is not an extremal point of $K_{A}$ and let $J=\left[P_{0}, Q\right]$. The segment $J$ intersects all the $D_{n}$ for $n \in \mathbb{N}$. If $P_{0}$ is in $T$ (and not in $\bar{T} \backslash T$ ) then, as $T$ is mixing, there exist $u_{1}, \ldots, u_{r} \in \mathbb{F}_{N}$ such that

$$
J \subseteq u_{1} I \cup \cdots \cup u_{r} I .
$$

The partial isometries $u_{1}, \ldots, u_{r}$ are not empty and for each $k, u_{k} I \cap K_{A}=I u_{k}^{-1}$ thus, using Proposition 5.5,

$$
J \subseteq I u_{1}^{-1} \cup \cdots \cup I u_{r}^{-1} .
$$

Hence, for $n$ bigger than all the lengths of the $u_{i}, J \cap D_{n}$ is empty. A contradiction.

Thus, $P_{0}$ is in $\bar{T} \backslash T$. We get that $P_{0}$ is an extremal point of $\bar{E}_{0}$ and, as $E_{0}$ is open in $K_{A}, P_{0}$ is in $\bar{E}_{0}-E_{0}$. As $P_{0}$ is not in $E_{0}$ there are at least two partial isometries $a, b \in A^{ \pm 1}$ defined at $P_{0}$. One at least of $a$ and $b$ is not defined in $E_{0}$, say $a$, and thus is defined only at $P_{0}$. Thus, in $T$, there are at least two directions going out from $P_{0}$ (one containing $K_{A}$ and the other containing $a^{-1} K_{A}$ ). This contradicts the fact that $P_{0}$ is in $\bar{T} \backslash T$.

Corollary 5.15. Let $T$ be an $\mathbb{R}$-tree with a mixing action of $\mathbb{F}_{N}$ by isometries. Let $\Omega$ be the limit set of $T$. The following are equivalent:

(1) $T$ is of surface type.

(2) $\Omega_{A}$ has finitely many components (i.e. $\Omega_{A}$ is a forest).

(3) $\Omega$ is connected.

(4) $\Omega$ contains $T$, that is to say $T \subseteq \Omega \subseteq \bar{T}$.

Proof. The equivalence of conditions 2 and 1 follows from the definition of surface type. Conditions 3 and 4 are equivalent because $T$ is minimal. We proved in Proposition 5.13 that condition 1 implies condition 3. From the dichotomy of Proposition 5.14 and from Theorem 5.11 we get that condition 3 implies condition 1

\section{Botanic memo}

In this section we give a glossary of our classification of trees for the working mathematician. 
Let $T$ be an $\mathbb{R}$-tree with a minimal, very small action of $\mathbb{F}_{N}$ by isometries with dense orbits. We assume that the action is indecomposable or at least that $T$ is not of mixed type.

For a basis $A$ of $\mathbb{F}_{N}, \Omega_{A}$ is the relative limit set and $K_{A}=\operatorname{conv}\left(\Omega_{A}\right)$ is the compact heart. The compact heart $K_{A}$ is either a finite tree (and in the good cases an interval) or not. This dichotomy is a property of $T$ and does not depend of the choice of a particular basis $A$ of $\mathbb{F}_{N}$.

As we assumed that $T$ is indecomposable, $\Omega_{A}$ is either a compact forest, that is to say it has finitely many connected components (and in the good cases $\Omega_{A}=K_{A}$ is a tree) or totally disconnected (and in the good cases a Cantor set). This dichotomy is a property of $T$ and does not depend on the choice of a particular basis $A$ of $\mathbb{F}_{N}$.

The limit set of $T$ is $\Omega=Q^{2}(L(T))=\mathbb{F}_{N} \cdot \Omega_{A}$. It is either totally disconnected (if $\Omega_{A}$ is), or it is connected (if $\Omega_{A}$ is a forest): in the later case, $\Omega$ is a tree, $T \subseteq \Omega \subseteq \bar{T}$.

For such a tree $T$ we considered two indices: the geometric index $\operatorname{ind}_{\text {geo }}(T)$ and the $Q$-index $\operatorname{ind}_{\mathcal{Q}}(T)$. Both indices are bounded above by $2 N-2$. We sum up the terminology for $T$ and the results of Section 5.7 in the following table.

\begin{tabular}{|c|c|c|c|}
\hline & geometric & not geometric \\
\hline & & $\begin{array}{c}K_{A} \text { is a finite tree } \\
\text { 业 } \\
\operatorname{ind}_{\text {geo }}(T)=2 N-2\end{array}$ & $\begin{array}{l}K_{A} \text { is not a finite tree } \\
\operatorname{ind}_{\text {geo }}(T)<2 N-2\end{array}$ \\
\hline 芯 & $\begin{array}{l}\Omega \text { is a tree }(T \subset \Omega) \\
\Omega_{A} \text { is a finite forest } \\
\operatorname{ind}_{\mathscr{Q}}(T)=2 N-2\end{array}$ & surface & pseudo-surface \\
\hline  & $\begin{array}{c}\Omega \text { is totally disconnected } \\
\mathbb{1} \\
\Omega_{A} \text { is totally disconnected } \\
\\
\operatorname{ind}_{Q}(T)<2 N-2\end{array}$ & Levitt & pseudo-Levitt \\
\hline
\end{tabular}

\section{References}

[Bes02] M. Bestvina, $\mathbb{R}$-trees in topology, geometry, and group theory. In Handbook of geometric topology, North-Holland, Amsterdam 2002, 55-91. Zbl 0998.57003 MR 1886668 
[BF95] M. Bestvina and M. Feighn, Outer limits. Unpublished, 1994. http://andromeda.rutgers.edu/ feighn/papers/outer.pdf.

[BF95] M. Bestvina and M. Feighn, Stable actions of groups on real trees. Invent. Math. 121 (1995), 287-321. Zbl 0837.20047 MR 1346208

[CH10] T. Coulbois and A. Hilion, Botany of irreducible automorphisms of free groups. Pacific J. Math. 256 (2012), no. 2, 291-307. Zbl 1259.20031 MR 2944977

[CL89] M. M. Cohen and M. Lustig, On the dynamics and the fixed subgroup of a free group automorphism. Invent. Math. 96 (1989), 613-638. Zbl 0678.57001 MR 996557

[CL95] M. M. Cohen and M. Lustig, Very small group actions on $\mathbb{R}$-trees and Dehn twist automorphisms. Topology 34 (1995), 575-617. Zbl 0844.20018 MR 1341810

[CHL07] T. Coulbois, A. Hilion, and M. Lustig, Non-unique ergodicity, observers' topology and the dual algebraic lamination for $\mathbb{R}$-trees. Illinois J. Math. 51 (2007), 897-911. Zbl 1197.20020 MR 2379729

[CHL08] T. Coulbois, A. Hilion, and M. Lustig, $\mathbb{R}$-trees and laminations for free groups II: the dual lamination of an $\mathbb{R}$-tree. J. London Math. Soc. (2) 78 (2008), 737-754. Zbl 1198.20023 MR 2456902

[CHL09] T. Coulbois, A. Hilion, and M. Lustig, $\mathbb{R}$-trees, dual laminations and compact systems of partial isometries. Math. Proc. Cambridge Philos. Soc. 147 (2009), 345-368. Zbl 1239.20030 MR 2525931

[CHR11] T. Coulbois, A. Hilion, and P. Reynolds, Indecomposable $F_{N}$-trees and minimal laminations. Groups. Geom. Dyn., to appear; arXiv:1110.3506

[CV86] M. Culler and K. Vogtmann, Moduli of graphs and automorphisms of free groups. Invent. Math. 84 (1986), 91-119. Zbl 0589.20022 MR 830040

[FLP79] A. Fathi, F. Laudenbach, and V. Poénaru (eds.), Travaux de Thurston sur les surfaces. Astérisque 66-67 (1979). Zbl 0406.00016 MR 0568308

[Gab96] D. Gaboriau, Dynamique des systèmes d'isométries: sur les bouts des orbites. Invent. Math. 126 (1996), 297-318. Zbl 0880.58009 MR 1411135

[Gab97] D. Gaboriau, Générateurs indépendants pour les systèmes d'isométries de dimension un. Ann. Inst. Fourier (Grenoble) 47 (1997), 101-122. Zbl 0861.20030 MR 1437180

[GJLL98] D. Gaboriau, A. Jaeger, G. Levitt, and M. Lustig, An index for counting fixed points of automorphisms of free groups. Duke Math. J. 93 (1998), 425-452. Zbl 0946.20010 MR 1626723

[GL95] D. Gaboriau and G. Levitt, The rank of actions on R-trees. Ann. Sci. École Norm. Sup. (4) 28 (1995), 549-570. Zbl 0835.20038 MR 1341661

[GLP94] D. Gaboriau, G. Levitt, and F. Paulin, Pseudogroups of isometries of $\mathbb{R}$ and Rips' theorem on free actions on $\mathbb{R}$-trees. Israel J. Math. 87 (1994), 403-428. Zbl 00645166 MR 1286836

[Ger83] S. M. Gersten, Intersections of finitely generated subgroups of free groups and resolutions of graphs. Invent. Math. 71 (1983), 567-591. Zbl 0521.20014 MR 695907

[Gui05] V. Guirardel, Cœur et nombre d'intersection pour les actions de groupes sur les arbres. Ann. Sci. École Norm. Sup. (4) 38 (2005), 847-888. Zbl 1110.20019 MR 2216833 
[Gui08] V. Guirardel, Actions of finitely generated groups on $\mathbb{R}$-trees. Ann. Inst. Fourier (Grenoble) 58 (2008), 159-211. Zbl 1187.20020 MR 2401220

[HM07] M. Handel and L. Mosher, Parageometric outer automorphisms of free groups. Trans. Amer. Math. Soc. 359 (2007), 3153-3183. Zbl 1120.20042 MR 2299450

[Ima76] H. Imanishi, Structure of codimension one foliations which are almost without holonomy. J. Math. Kyoto Univ. 16 (1976), 93-99. Zbl 0335.57019 MR 0415636

[Kap01] M. Kapovich, Hyperbolic manifolds and discrete groups. Progr. Math. 183, Birkhäuser, Boston 2001. Zbl 0958.57001 MR 1792613

[Lev93] G. Levitt, La dynamique des pseudogroupes de rotations. Invent. Math. 113 (1993), 633-670. Zbl 0791.58055 MR 1231840

[LL03] G. Levitt and M. Lustig, Irreducible automorphisms of $F_{n}$ have north-south dynamics on compactified outer space. J. Inst. Math. Jussieu 2 (2003), 59-72. Zbl 1034.20038 MR 1955207

[LL08] G. Levitt and M. Lustig, Automorphisms of free groups have asymptotically periodic dynamics. J. Reine Angew. Math. 619 (2008), 1-36. Zbl 1157.20017 MR 2414945

[Mor88] J. W. Morgan, Ergodic theory and free actions of groups on $\mathbb{R}$-trees. Invent. Math. 94 (1988), 605-622. Zbl 0676.57001 MR 969245

[Rey10] P. Reynolds, On indecomposable trees in the boundary of outer space. Geom. Dedicata 153 (2011), 59-71. Zbl 1245.20029 MR 2819663

[Sta83] J. R. Stallings, Topology of finite graphs. Invent. Math. 71 (1983), 551-565. Zbl 0521.20013 MR 695906

[Vog02] K. Vogtmann, Automorphisms of free groups and outer space. Geom. Dedicata 94 (2002), 1-31. Zbl 1017.20035 MR 1950871

Received January 4, 2012; revised May 9, 2012

T. Coulbois, I2M, Centre de Mathématiques et Informatique (CMI), Aix-Marseille Université (AMU), 39, rue F. Joliot Curie, 13453 Marseille Cedex 13, France

E-mail: thierry.coulbois@univ-amu.fr

A. Hilion, I2M, Centre de Mathématiques et Informatique (CMI), Aix-Marseille Université (AMU), 39, rue F. Joliot Curie, 13453 Marseille Cedex 13, France

E-mail: arnaud.hilion@univ-amu.fr 